\title{
INFRARED PHOTOMETRY OF METAL RICH GALACTIC GLOBULAR CLUSTERS
}

\author{
Judith G. COHEN AND Celeste SLeEPER \\ Palomar Observatory, California Institute of Technology, Mail Code 105-24, Pasadena, California 91125 \\ Electronic mail: jlc@astro.caltech.edu, ecs@stro.caltech.edu \\ Received 1994 July 1; revised 1994 September 7
}

\begin{abstract}
Infrared photometry is presented for the giant branches of five galactic globular clusters, including three very metal rich ones located near the galactic center. New visual photometry was required for three of these objects. The metallicities of these clusters as inferred from the dereddened colors of their giant branches are in reasonable agreement with those inferred by spectroscopy of the integrated light of these systems. The spread in color seen along the giant branches of the most highly reddened of these globular clusters is shown to be consistent with patchy interstellar reddening. Abundance variations within a cluster are not required to explain the observations. The range of metallicity is confined to less than 0.2 dex in NGC 5927, and to 0.1 dex in NGC 7099. The spread in $V-K$ color is a strong constraint on abundance variations within a globular cluster.
\end{abstract}

\section{INTRODUCTION}

The very metal rich galactic globular clusters comprise a group of poorly studied objects. They often have high foreground reddening, suffer severe crowding because they are located near the galactic center, and may have serious field star contamination. In many cases, the only available optical photometry is old photographic photometry. We have obtained new infrared photometry using an array detector of the giant branch stars in several of these clusters (and in two cases, new optical photometry as well) with the following goals. First we would like to confirm, based on the location of their giant branches, the estimates of the high metallicity of these clusters. Second we would like to determine whether the large apparent scatter in the existing optical color magnitude diagrams is due to poor photometry, an internal metallicity dispersion, or reddening variations. We would also like to check on the luminosity of the brightest red giant, which defines the luminosity of the helium flash and can be used as a check on the cluster distance. Finally, we would like to analyze the luminosity variances of these globular clusters in the infrared.

We have picked a sample of galactic globular clusters with metallicities at least twice that of 47 Tuc based on the compendium of Zinn \& West (1984), with $\mathrm{E}(B-V) \leqslant 0.6$ mag, a distance not larger than $10 \mathrm{kpc}$, and $v_{r}$ significantly different from $0 \mathrm{~km} \mathrm{~s}^{-1}$, so that if necessary, field stars can easily be separated from cluster members using radial velocity measurements. The three program clusters are NGC 5927, NGC 6624, and NGC 6528. Two other clusters, NGC 6171 and NGC 7099, were also observed in the infrared. As initial values to guide us, we use the metallicities of Zinn \& West (1984), updated by Armandroff \& Zinn (1988). We use the values of $\mathrm{E}(B-V)$ and apparent visual brightness of the horizontal branch at the color of the RR Lyrae gap tabulated by Peterson (1993).

\section{OBSERVATIONS}

The advantages of using an infrared array detector for investigation of the giant branch of highly reddened galactic globular clusters are obvious. Davidge \& Simons (1991), who studied M69 in this way, review the major points. See also Davidge et al. (1992). For our observations, we have used the Rockwell Nicmos 3 infrared array camera described in Persson et al. (1992) at the Cassegrain focus of the 40 in. Swope telescope at the Las Campanas Observatory in Chile for five nights in June 1992 . This array has $256 \times 256$ pixels, covering $0.45 \mathrm{arcsec} / \mathrm{pixel}$ or a square 116 arcsec on a side. The seeing at $K$ was 1.0 to 1.5 arcsec. Observations were made at $J$ and at $K$ only. The fainter standards of Elias $e t a l$. (1982) were used. Multiple short exposures (60 and $120 \mathrm{~s}$ at $J$, and 30 and $60 \mathrm{~s}$ at $K$ ) were obtained for the globular cluster fields, typically with six images at each exposure time taken at each positional setting. Every field was observed at three positional settings, each slightly offset from the others on the sky, to help minimize variations arising from flatfielding problems. Two different fields were observed in most of these globular clusters. These were chosen to avoid the extreme crowding of the core of the cluster, while not being so far from the center that field star contamination becomes serious.

We slightly modified IRAF scripts originally written by Eric Persson to linearize the frames, collect the multiple exposures at each position, remove the dark signal, flatten the data using dome flats, process the standard stars, and make mosaics of the globular cluster data in $J$ and in $K$. Once this was accomplished, we reversed to using FIGARO as the data analysis package. DAOPHOT (Stetson 1987) was used to determine magnitudes from each of the three positional settings for each field. The $\sigma$ values given here are those of the multiple measurements of $J$ or $K$ magnitude for each object; they are not computed from the signal and sky values for a measured magnitude.

Saturation of the central pixel of stellar images occurs on 
TABLE 1. $V$ and $R$ photometry for NGC 5927.

\begin{tabular}{|c|c|c|c|c|c|c|c|c|c|c|c|}
\hline$\underset{\text { (pixels) }}{\mathbf{x}}$ & $\underset{\text { (pixels) }}{\mathbf{y}}$ & Star ID & $\underset{\text { (mag) }}{\text { V(CTIO) }}$ & $\underset{\text { (mag) }}{\Delta(\mathrm{V})}$ & $\begin{array}{c}\text { V-R } \\
\text { (mag) }\end{array}$ & $\begin{array}{c}x \\
\text { (pixels) }\end{array}$ & $\underset{\text { ixels) }}{\mathbf{y}}$ & Star ID & $\frac{\text { (mag) }}{\mathrm{V}(\mathrm{CTIO})}$ & $\begin{array}{c}\Delta(\mathrm{V}) \\
(\mathrm{mag})\end{array}$ & $\begin{array}{l}\mathrm{V}-\mathrm{R} \\
\text { (mag) }\end{array}$ \\
\hline 345 & 123 & & 15.53 & & 0.47 & 524 & 124 & & 15.30 & & 1.07 \\
\hline 180 & 146 & $1-436$ & 17.19 & 0.03 & 0.84 & 204 & 148 & $2-873$ & 16.41 & 0.04 & 0.98 \\
\hline 276 & 148 & $2-884$ & 16.08 & -0.10 & 0.71 & 452 & 154 & & 17.13 & & 0.78 \\
\hline 104 & 156 & $1-445$ & 16.13 & -0.43 & 0.60 & 156 & 162 & $1-439$ & 16.54 & 0.02 & 0.82 \\
\hline 484 & 166 & & 16.94 & & 0.84 & 111 & 169 & $1-447$ & 16.57 & -0.23 & 0.80 \\
\hline 264 & 171 & $2-888$ & 16.59 & 0.02 & 0.79 & 113 & 177 & $1-448$ & 16.44 & -0.25 & 0.92 \\
\hline 265 & 177 & $2-889$ & 17.17 & 0.05 & 0.72 & 312 & 177 & $2-886$ & 15.02 & 0.01 & 1.13 \\
\hline 106 & $179^{\circ}$ & $1-449$ & 17.02 & -0.35 & 0.81 & 335 & 183 & $3-907$ & 16.75 & 0.11 & 0.86 \\
\hline 159 & 185 & $1-463$ & 16.58 & -0.08 & 0.77 & 127 & 194 & & 16.62 & & 0.91 \\
\hline 190 & 194 & & 15.42 & & 0.98 & 169 & 196 & & 16.15 & & 0.76 \\
\hline 184 & 199 & $1-467$ & 17.01 & -0.05 & 0.78 & 192 & 206 & $1-468$ & 16.33 & -0.04 & 0.87 \\
\hline 390 & 210 & $3-904$ & 16.72 & 0.00 & 0.80 & 106 & 214 & $1-459$ & 16.61 & -0.25 & 0.83 \\
\hline 129 & 213 & $1-476$ & 16.54 & -0.18 & 0.80 & 288 & 213 & $2-892$ & 15.68 & -0.02 & 0.80 \\
\hline 142 & 215 & $1-474$ & 17.12 & -0.13 & 0.79 & 298 & 215 & $2-893$ & 17.10 & 0.08 & 0.78 \\
\hline 460 & 216 & & 13.27 & & 0.76 & 440 & 219 & $3-902$ & 17.32 & 0.07 & 0.80 \\
\hline 153 & 225 & $1-472$ & 15.87 & -0.29 & 0.98 & 145 & 227 & $1-473$ & 14.74 & -0.23 & 1.26 \\
\hline 231 & 229 & $2-891$ & 15.05 & -0.21 & 0.98 & 117 & 229 & $1-477$ & 16.59 & -0.19 & 0.80 \\
\hline 176 & 233 & $1-471$ & 17.28 & -0.07 & 0.86 & 286 & 233 & $2-897$ & 16.46 & 0.09 & 0.76 \\
\hline 96 & 234 & $1-479$ & 16.52 & -0.43 & 0.80 & 469 & 234 & & 15.06 & & 0.42 \\
\hline 490 & 235 & & 16.01 & & 0.57 & 526 & 235 & & 14.94 & & 0.71 \\
\hline 374 & 236 & $3-1294$ & 14.81 & 0.03 & 0.96 & 216 & 244 & $2-501$ & 15.27 & -0.06 & 0.48 \\
\hline 111 & 246 & $1-5$ & 16.62 & -0.17 & 0.77 & 98 & 248 & $1-1$ & 16.85 & -0.27 & 0.82 \\
\hline 472 & 251 & & 15.67 & & 0.67 & 186 & 252 & $1-10$ & 16.68 & 0.00 & 0.81 \\
\hline 105 & 253 & $1-3$ & 16.52 & -0.27 & 0.79 & 114 & 258 & $1-4$ & 15.01 & -0.65 & 0.79 \\
\hline 490 & 258 & & 15.60 & & 0.50 & 97 & 261 & $1-2$ & 16.19 & -0.55 & 0.84 \\
\hline 156 & 269 & $1-23$ & 15.47 & -0.20 & 1.05 & 195 & 271 & $1-17$ & 15.94 & -0.09 & 0.82 \\
\hline 253 & 279 & $2-516$ & 16.56 & 0.01 & 0.80 & 86 & 282 & & 16.08 & & 0.74 \\
\hline 393 & 285 & $3-1287$ & 17.26 & -0.01 & 0.85 & 55 & 288 & & 17.15 & & 0.86 \\
\hline 216 & 289 & & 16.07 & & 0.82 & 109 & 289 & & 15.36 & & 0.94 \\
\hline 170 & 288 & $1-21$ & 17.18 & -0.06 & 0.83 & 254 & 289 & $2-517$ & 16.61 & -0.04 & 0.80 \\
\hline 93 & 289 & & 16.71 & & 0.83 & 127 & 293 & $1-29$ & 16.71 & -0.17 & 0.83 \\
\hline 176 & 298 & $1-18$ & 16.58 & -0.18 & 0.75 & 259 & 297 & $2-519$ & 16.84 & -0.01 & 0.77 \\
\hline 70 & 299 & & 16.20 & & 0.81 & 75 & 300 & & 16.82 & & 0.84 \\
\hline 96 & 301 & $1-33$ & 16.27 & -0.34 & 0.81 & 206 & 301 & & 16.44 & & 0.80 \\
\hline 225 & 303 & $2-522$ & 16.48 & -0.03 & 0.79 & 247 & 304 & $2-521$ & 13.66 & -0.25 & 0.92 \\
\hline 160 & 306 & $1-40$ & 15.76 & -0.26 & 0.91 & 53 & 308 & $2-021$ & 16.47 & -0.25 & $\begin{array}{l}0.92 \\
0.83\end{array}$ \\
\hline 66 & 308 & & 16.55 & & 0.80 & 167 & 308 & $1-41$ & 16.43 & -0.29 & 0.77 \\
\hline 469 & 308 & & 15.81 & & 0.90 & 193 & 311 & $1-44$ & 16.77 & -0.02 & 0.86 \\
\hline 247 & 304 & & 13.66 & & 0.92 & 518 & 311 & & 16.37 & & 0.58 \\
\hline 93 & 312 & & 16.04 & & 0.68 & 248 & 312 & & 16.31 & & 0.70 \\
\hline 124 & 313 & $1-36$ & 17.30 & -0.14 & 0.79 & 92 & 316 & & 16.05 & & 0.68 \\
\hline 64 & 318 & $1-\infty$ & 17.11 & & 0.83 & 224 & 317 & $2-523$ & 16.59 & -0.05 & 0.79 \\
\hline 181 & 320 & $1-43$ & 17.33 & -0.05 & 0.85 & 54 & 322 & & 16.65 & & 0.80 \\
\hline 435 & 322 & $3-1274$ & 15.96 & -0.06 & 0.85 & 74 & 323 & & 16.51 & & 0.76 \\
\hline 153 & 325 & $1-38$ & 15.64 & -0.21 & 0.90 & 61 & 328 & & 16.68 & & 0.82 \\
\hline 264 & 328 & $2-525$ & 16.55 & -0.04 & 0.78 & 413 & 328 & $3-1276$ & 15.54 & -0.02 & 0.76 \\
\hline 256 & 329 & $2-524$ & 16.61 & -0.07 & 0.79 & 348 & 330 & $3-1278$ & 16.31 & 0.02 & 0.65 \\
\hline 134 & 331 & $1-37$ & 15.83 & -0.06 & 0.92 & 262 & 336 & $2-530$ & 16.21 & -0.27 & 0.56 \\
\hline 180 & 337 & $1-49$ & 16.74 & 0.01 & 0.80 & 87 & 342 & $1-66$ & 16.43 & -0.35 & 0.78 \\
\hline 123 & 342 & $1-59$ & 16.70 & -0.12 & 0.79 & 78 & 343 & $1-67$ & 16.67 & -0.33 & \\
\hline 268 & 350 & $2-531$ & 16.36 & -0.03 & 0.77 & 102 & 351 & $1-65$ & 14.94 & -0.30 & 1.08 \\
\hline 73 & 352 & $1-68$ & 15.91 & -0.65 & 0.66 & 57 & 354 & & 16.45 & & 0.77 \\
\hline 90 & 355 & $1-69$ & 15.60 & -0.39 & 0.97 & 247 & 356 & $2-532$ & 14.73 & -0.14 & 1.22 \\
\hline 230 & 356 & $2-533$ & 15.16 & -0.05 & 0.83 & 119 & 357 & $1-61$ & 17.29 & -0.11 & 0.81 \\
\hline 134 & 360 & $1-56$ & 16.59 & -0.01 & 0.77 & 366 & 361 & $3-1269$ & 16.86 & .0 .05 & 0.84 \\
\hline 324 & 363 & $3-1267$ & 16.02 & 0.02 & 0.95 & 87 & 367 & $1-70$ & 16.99 & -0.20 & 0.85 \\
\hline 184 & 369 & $2-534$ & 16.85 & -0.06 & 0.85 & 251 & 373 & & 14.84 & -0.20 & 1.15 \\
\hline 218 & 374 & $2-536$ & 15.18 & 0.01 & 1.03 & 344 & 376 & $3-1268$ & 17.27 & 0.03 & 0.83 \\
\hline 263 & 379 & $2-540$ & 15.79 & -0.10 & 0.59 & 279 & 379 & $2-541$ & 16.66 & 0.05 & 0.78 \\
\hline 129 & 386 & & 15.42 & & 0.99 & 249 & 384 & $2-539$ & 15.20 & -0.23 & 1.10 \\
\hline 85 & 387 & $1-86$ & 15.55 & -0.38 & 0.98 & 238 & 390 & $2-537$ & 17.10 & 0.01 & 0.82 \\
\hline 116 & 392 & $1-74$ & 12.83 & -0.29 & 0.82 & 99 & 403 & $1-84$ & 15.56 & -0.21 & 0.96 \\
\hline 311 & 408 & $3-1265$ & 15.01 & 0.01 & 0.87 & 164 & 410 & $2-549$ & 16.70 & 0.04 & 0.91 \\
\hline 199 & 421 & $2-550$ & 16.85 & 0.05 & 0.87 & 123 & 422 & $1-77$ & 17.05 & & 0.79 \\
\hline 262 & 429 & $2-544$ & 16.73 & & 0.80 & 209 & 430 & & 16.77 & & 0.81 \\
\hline 94 & 432 & $1-111$ & 14.55 & & 0.91 & 282 & 444 & & 16.95 & & 0.76 \\
\hline 161 & 447 & $2-557$ & 16.56 & -0.01 & 0.76 & 68 & 450 & $V-5$ & 14.96 & & 1.62 \\
\hline 333 & 450 & $3-1254$ & 14.78 & -0.29 & 0.79 & 122 & 452 & $2-559$ & 16.65 & -0.05 & 0.78 \\
\hline 325 & 456 & $3-1253$ & 14.23 & -0.22 & 0.79 & 309 & 465 & & 16.78 & & 0.81 \\
\hline 142 & 467 & & 13.52 & & 0.87 & 56 & 475 & $V-6$ & 14.91 & & 1.53 \\
\hline 106 & 478 & $2-563$ & 15.94 & -0.07 & 0.95 & 139 & 482 & & 17.12 & & 0.76 \\
\hline 259 & 483 & $3-1248$ & 16.77 & 0.04 & 0.81 & 87 & 485 & $2-584$ & 16.51 & -0.26 & 0.69 \\
\hline 312 & 490 & $3-1244$ & 16.59 & 0.02 & 0.76 & 265 & 500 & $3-1246$ & 16.63 & 0.04 & 0.79 \\
\hline 136 & 506 & $2-571$ & 16.70 & & 0.77 & 79 & 510 & $2-582$ & 15.27 & -0.06 & 0.84 \\
\hline 315 & 512 & $3-1242$ & 16.67 & 0.05 & 0.75 & 215 & 515 & $3-1235$ & 16.65 & 0.10 & 0.83 \\
\hline 63 & 516 & $2-580$ & 16.52 & 0.00 & 0.81 & 183 & 516 & $2-569$ & 16.73 & & 0.76 \\
\hline 255 & 521 & $3-1238$ & 15.76 & 0.04 & 1.01 & 281 & 523 & & 16.53 & & 0.62 \\
\hline 126 & 533 & $2-573$ & 16.24 & & 0.81 & 171 & 541 & & 16.29 & & 0.90 \\
\hline 97 & 558 & & 16.65 & & 0.77 & 184 & 563 & & 15.67 & & 0.57 \\
\hline 72 & 570 & & 15.77 & & 0.97 & 90 & 580 & & 17.22 & & 0.74 \\
\hline 210 & 582 & & 13.00 & & 0.62 & & & & & & \\
\hline
\end{tabular}


the shortest $J$ frames at $\approx 10.0 \mathrm{mag}$ and for the shortest $K$ frames at $\approx 8.7 \mathrm{mag}$.

\section{INDIVIDUAL CLUSTERS}

\subsection{NGC 5927}

NGC 5927 is a very metal rich globular cluster located at a distance of $8 \mathrm{kpc}$ about $35^{\circ}$ from the galactic center in the galactic plane. We have adopted $\mathrm{E}(B-V)=0.46 \mathrm{mag}$. Visual photometry by Menzies (1974) established that the reddening was patchy. Our two fields are centered in the region which he described as "apparently uniformly reddened" with $\mathrm{E}(B-V)=0.52$ mag. To supplement this rather old visual photometry, CCD frames were obtained through the $V$ and $R$ filters at the Yale $1.0 \mathrm{~m}$ telescope at CTIO in July 1993 using service observing. The data were reduced in standard ways (FIGARO and DAOPHOT) described above and the results are given in Table 1. The major purpose of the Tololo photometry was to obtain additional $V$ magnitudes for stars observed in the infrared, but it also served to confirm the estimate of the magnitude level of the red horizontal branch, which was $16.55 \pm 0.08$ mag from the new data, and is given as 16.60 mag by Menzies (1974).

The globular cluster distance scale we are using assumes $M_{V}(\mathrm{HB})=0.16[\mathrm{Fe} / \mathrm{H}]+1.02$, from Jones et al. (1992). The variation of absolute magnitude of the RR Lyraes as a function of metallicity they deduce by collating all available Baade-Wesslink analyses of field RR Lyrae variables gives results comparable to other techniques. Various authors (Cohen 1992; Liu \& Janes 1990; for example) have demonstrated that globular cluster RR Lyrae variables follow the same relationships between absolute $V$ magnitude and metallicity or between absolute $K$ magnitude and period as have been demonstrated to hold for field stars. The exact choice of a globular cluster distance scale from among the various possibilities currently in fashion does not affect the results of this paper in a substantive way, since we are to first order comparing the measured parameters of the sample globular clusters against those of calibrating globular clusters whose properties are well determined.

Table 2 lists the measurements at $J$ and at $K$ for NGC 5927. The first two columns give $x$ and $y$ in pixels, where positive $y$ corresponds (approximately) to north and positive $x$ to west. Then follow the observed $J$ and $K$ magnitudes, with dispersions given if there was more than one measurement. The dispersions between infrared magnitudes of a star as measured on multiple frames are significantly larger than those predicted purely from the statistics of the photometry on any individual frame. The final columns give the optical identification number from Menzies (1974), if optical photometry is available for that star.

Because of our small fields, we have only six stars in common with the early study of Frogel et al. (1983a), who observed the brightest and reddest stars over the entire cluster based on the Menzies (1974) photometry. Ignoring two variable stars, for the four remaining stars in common, we find a systematic difference of $0.03 \pm 0.02 \mathrm{mag}$ in $J$ and 0.08 $\pm 0.04 \mathrm{mag}$ in $K$, with our magnitudes being fainter.
Kuchinski et al. (1994) also obtained new infrared photometry for this cluster with the $2.5 \mathrm{~m}$ telescope at Las Campanas Observatory. We have 43 stars in common, and ignoring one with a large discrepancy which is probably affected by crowding, the mean difference in the $J-K$ color is 0.024 mag, with $\sigma=0.10 \mathrm{mag}$, while the mean difference in the $K$ magnitudes is -0.001 , with $\sigma=0.12 \mathrm{mag}$. This is a very encouraging comparison of two independent data sets.

Figures 1(a) and 1(b) show the $J-K$ and $V-K$ colors as a function of $K$ magnitude. Open circles denote stars without optical photometry, while filled circles denote those stars with optical photometry. The two brightest stars in the infrared denoted by open triangles are the extremely red variables V5 and V6. In an optical color-magnitude diagram, the upper end of the giant branch, particularly the red variables, would turn over towards fainter magnitudes for the reddest stars (see, for example, Ortolani et al. 1991b,a), while this does not happen in Fig. 1. The same phenomenon occurs to an even greater extent in NGC 6624 and also in NGC 6553 (Davidge \& Simons 1994), both very metal rich globular clusters. We believe it is due to the anomalous colors and unexpected faintness at visual wavelengths of very metal rich cool giants due to large molecular blanketing.

The clump of stars somewhat blueward of the giant branch of NGC 5927 at $K_{0} \approx 13.1 \mathrm{mag}$ is presumably the horizontal branch. This feature is much clearer in Kuchinski et al.'s data.

\section{$3.2 N G C 6528$}

NGC 6528 is a very metal rich globular cluster located in Baade's window near the galactic center. The Zinn \& West (1984) metallicity of $[\mathrm{Fe} / \mathrm{H}]=+0.12 \mathrm{dex}$ is significantly larger than that of Armandroff \& Zinn (1988), who give reasons why the latter value is to be preferred. $B, V$ visual photometry is given by van den Bergh \& Younger (1979). CCD photometry at $B, V, R$, and $I$ is given by Ortolani et al. (1991b). Although Ortolani et al. (1991a,b) did not publish the details of their measurements, they kindly sent them to us. Ortolani et al. deduce $\mathrm{E}(B-V)=0.55 \mathrm{mag}$, with differential reddening of up to $\Delta \mathrm{E}(B-V)=0.33$ mag across the face of the cluster, and a distance of $7.5 \mathrm{kpc}$. Note that their measured location of the horizontal branch is $\approx 0.4 \mathrm{mag}$ fainter than that obtained by van den Bergh \& Younger (1979), where the HB was near the limit of their photometry. Using our assumed $M_{V}(\mathrm{HB})$ with Ortolani et al.'s $V_{\mathrm{HB}}=17.1$ mag, we deduce a distance modulus of 14.42 mag.

The cluster is confined to a small field on the sky. Thus, a single field centered on the globular cluster was observed on two different nights. A background field 400 arcsec to the east of the cluster center was observed on the same two nights. Table 3 lists the measurements of $J$ and at $K$ for NGC 6528. The format is identical to Table 2. The identifications are from van den Bergh \& Younger (1979), or if not included there, from Ortolani's list. The $V$ magnitudes are from Ortolani's list. There is no previous infrared photometry for any stars in this globular cluster.

Figures 2(a) and 2(b) show the $J-K$ and $V$ - $K$ colors as a function of $K$ magnitude. Open circles denote stars without 


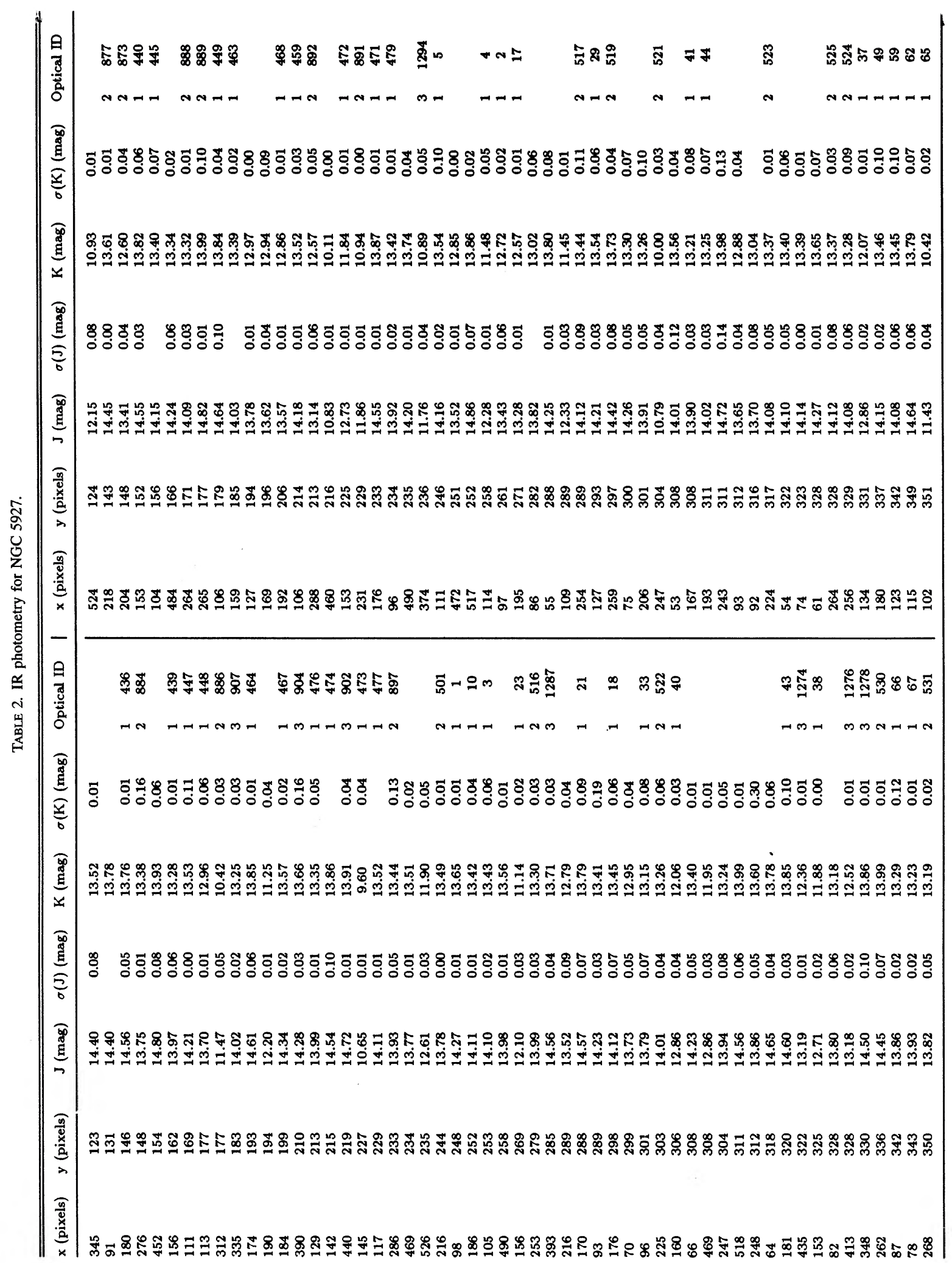




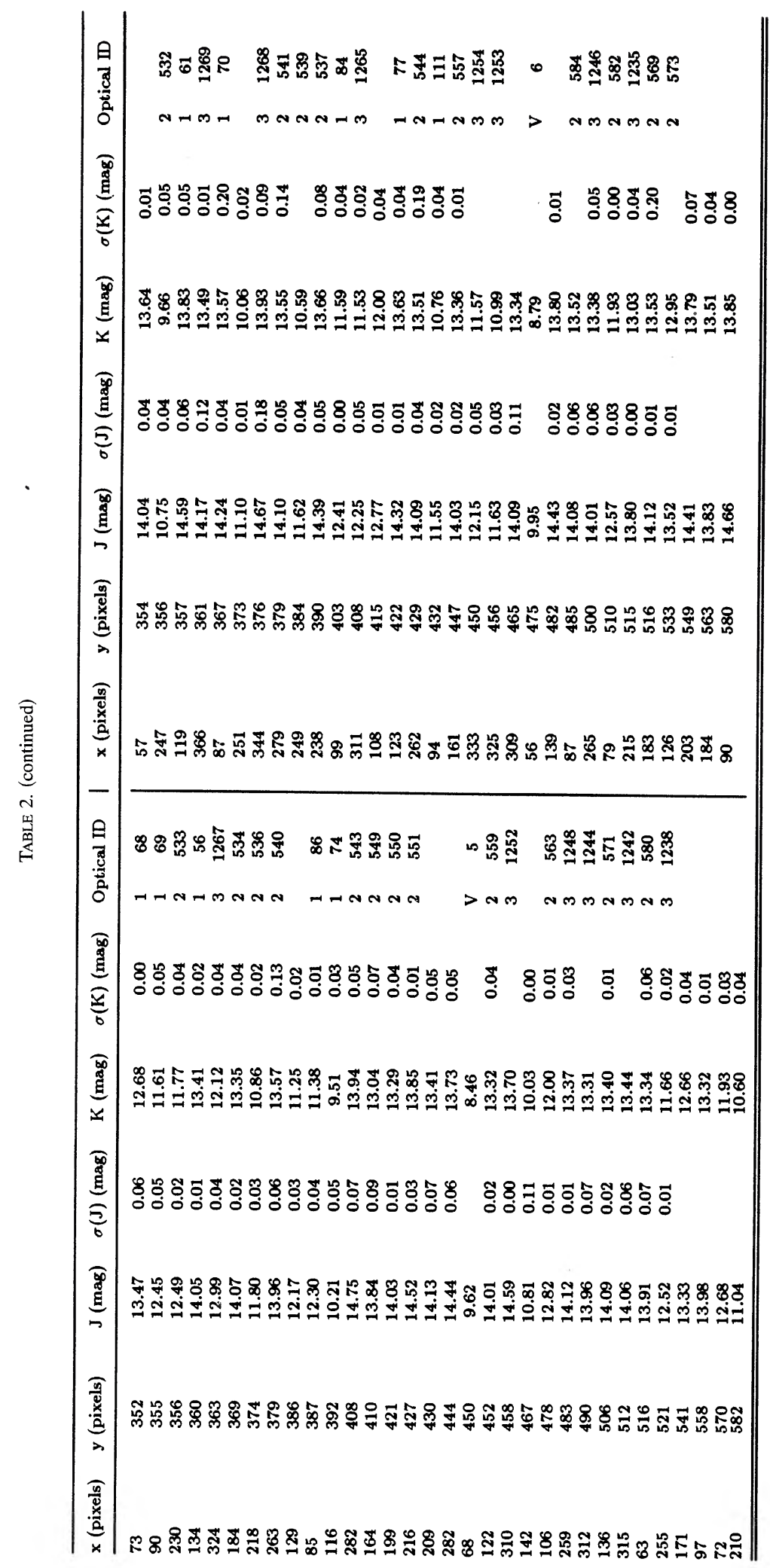



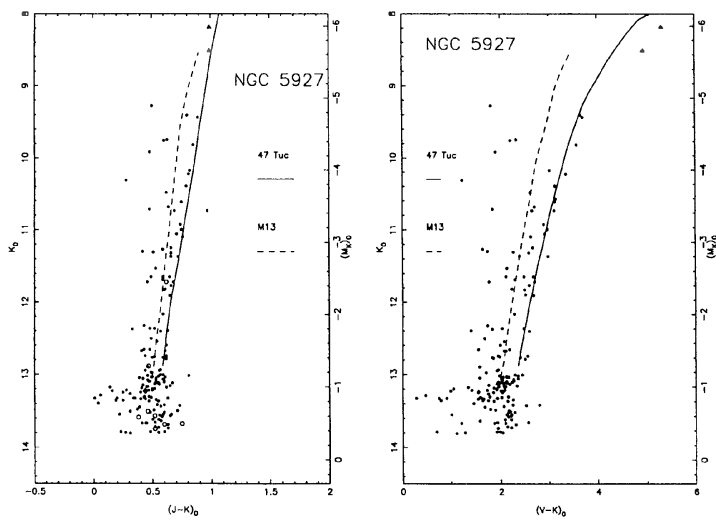

Fig. 1. (a) and (b) show the $J-K$ and $V-K$ colors as a function of reddening-corrected $K$ magnitude for the giants in NGC 5927. The open symbols in (a) denote stars for which no visual photometry is available. The two open triangles indicate two very red variables near the tip of the giant branch.

optical photometry, while filled circles denote those stars with optical photometry (the majority of the objects). In the infrared color-magnitude diagram, unlike in corresponding optical color-magnitude diagrams, the stars progress smoothly and monotonically brighter as they become redder. This phenomenon was discussed in Sec. 3.1.

\section{$3.3 N G C 6624$}

NGC 6624 is a small globular cluster located close to the galactic center at a distance of $6.9 \mathrm{kpc}$. We have adopted $\mathrm{E}(B-V)=0.28 \mathrm{mag}$ for this object. Photographic photometry is available from Liller \& Carney (1978). Its metallicity is believed to be comparable to that of 47 Tuc, i.e., significantly less than that of NGC 5927 or NGC 6528. Three fields were observed on three different nights whose centers are $\approx 80$ arcsec east, west, and south of the center of the globular cluster. Table 4 lists the measurements at $J$ and at $K$ for NGC 6624. The format is identical to Table 2 . There is no previous infrared photometry for any stars in this globular cluster. The optical identifications are from Liller \& Carney (1978). To supplement this rather old visual photometry, CCD frames were obtained through the $V$ and $R$ filters at the Yale $1.0 \mathrm{~m}$ telescope at CTIO in July 1993 using service observing. The data were reduced in standard ways (FIGARO and DAOPHOT) described above and the resulting magnitudes are listed in Table 5. The major purpose of the Tololo photometry was to obtain additional $V$ magnitudes for stars observed in the infrared, but it also served to confirm the estimate of the magnitude level of the red horizontal branch, which is 15.98 $\pm 0.08 \mathrm{mag}$ from the new data, and is given as 16.05 mag by Liller \& Carney (1978).

Figures 3(a) and 3(b) show the $J-K$ and $V-K$ colors as a function of $K$ magnitude. Open circles denote stars without optical photometry, while filled circles denote those stars with optical photometry. There is some contamination by field stars, but the general morphology of the giant branch is obvious. The red horizontal branch appears at $V \approx 16 \mathrm{mag}$

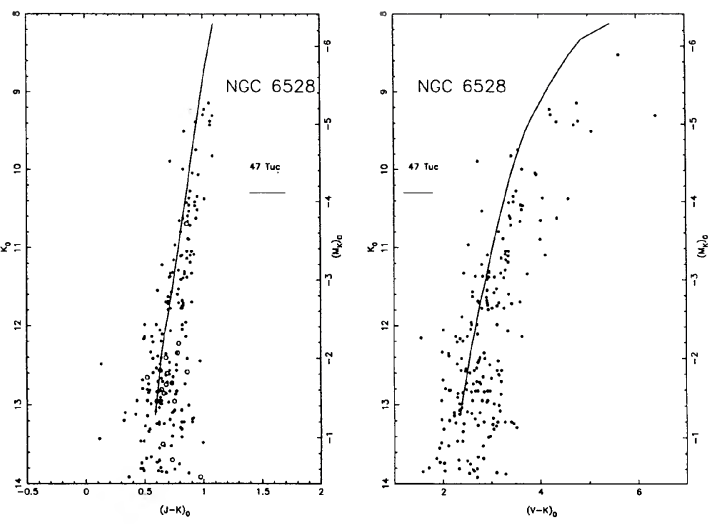

FIG. 2. The same as Figs. 1(a) and 1(b) for NGC 6528.

(Liller \& Carney 1978), and is apparent in Fig. 3 as the group of stars with $K_{0} \approx 13.2 \mathrm{mag}$.

\section{$3.4 N G C 6171$}

NGC 6171 is a moderately metal poor globular cluster in the direction of the galactic center but at a galactic latitude of $23^{\circ}$ with a fairly large reddening. We have adopted $\mathrm{E}(B-V)$ $=0.33 \mathrm{mag}$. Optical photometry was given by Sandage \& Katem (1964), while photometry reaching the main sequence was given by DaCosta et al. (1984). We covered several fields in the outer parts of the cluster from Las Campanas. Unfortunately, that night was not photometric, and the data were calibrated by repeating smaller fields in the outer parts of the cluster, and adding a field near the center with the infrared array on the $5 \mathrm{~m}$ Hale telescope at Palomar Mountain.

We use the numbering system of both Sandage \& Katem (1964) (denoted "1") and that of DaCosta et al. (1984) (denoted " 2 "). Infrared magnitudes for the program stars are given in Table 6, while Figs. 4(a) and 4(b) show the $J-K$ and $V-K$ colors as a function of $K$ magnitude. The symbols are as before, with the addition of "* ", which denotes an RR Lyrae variable, and of an open star, used for two stars which are definitely field stars based on their position in the $V$,

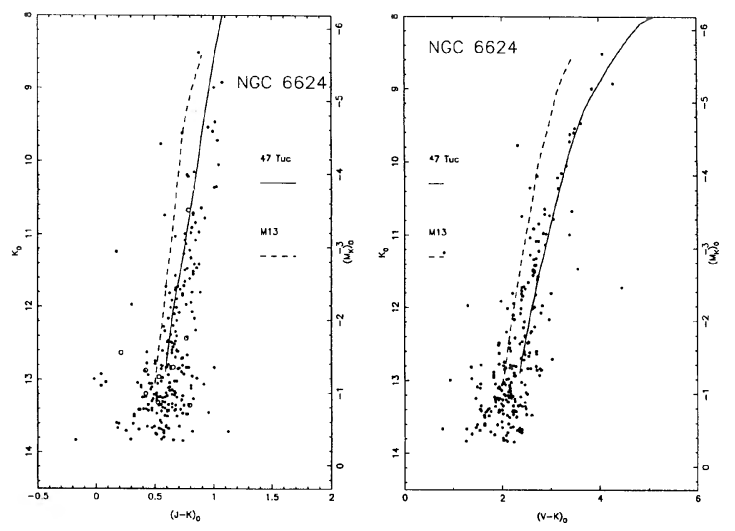

FIG. 3. The same as Figs. 1(a) and 1(b) for NGC 6624. 


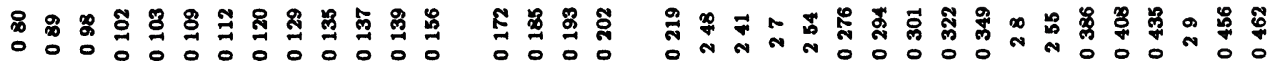

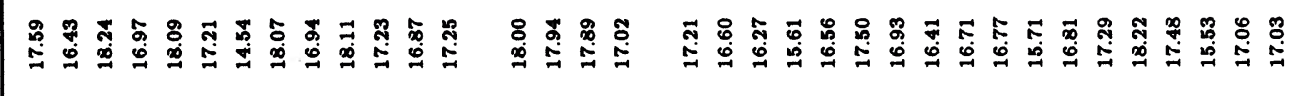

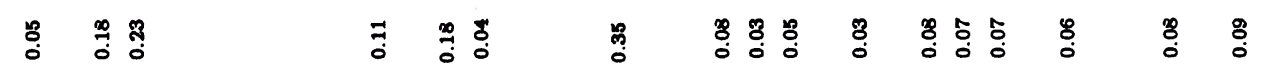

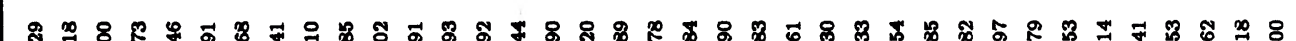

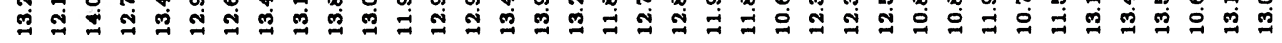

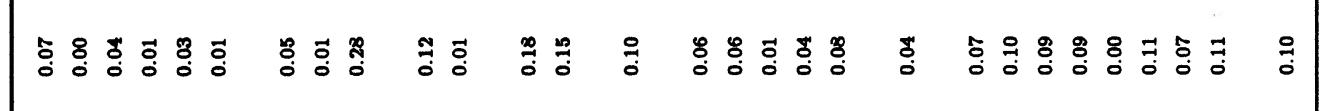

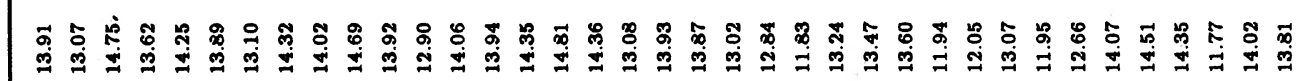

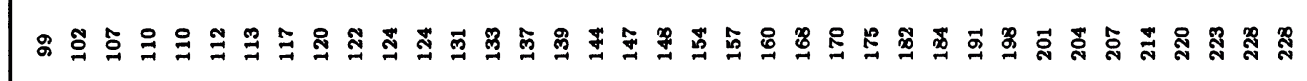
拿

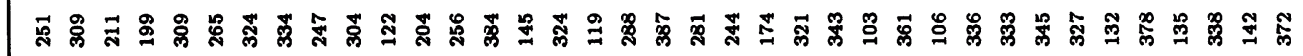

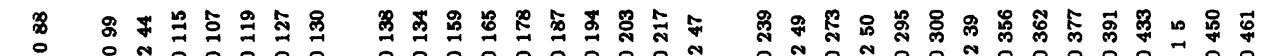

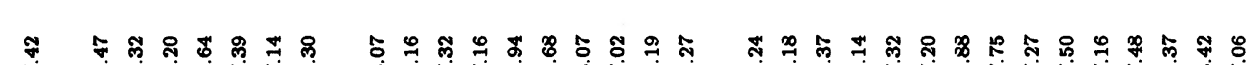

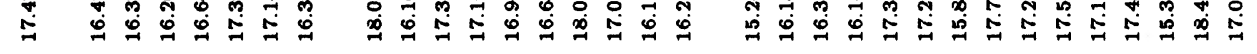

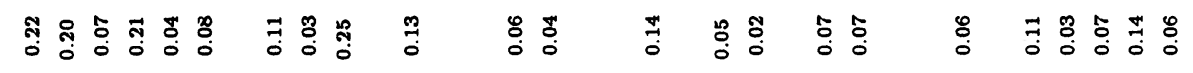

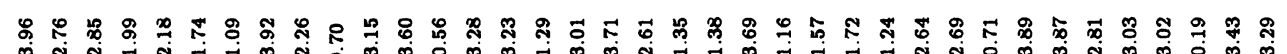
包

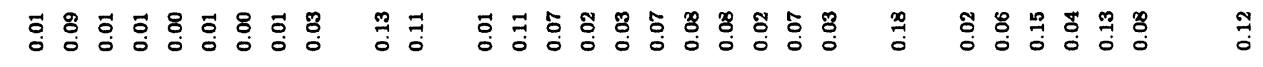

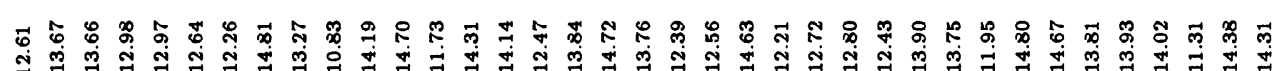

\& $\frac{\sqrt{6}}{\frac{x}{2}}$

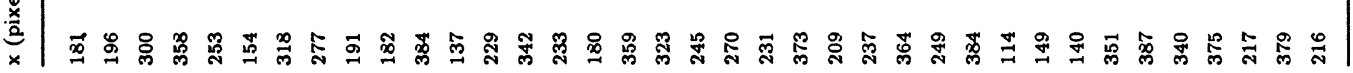




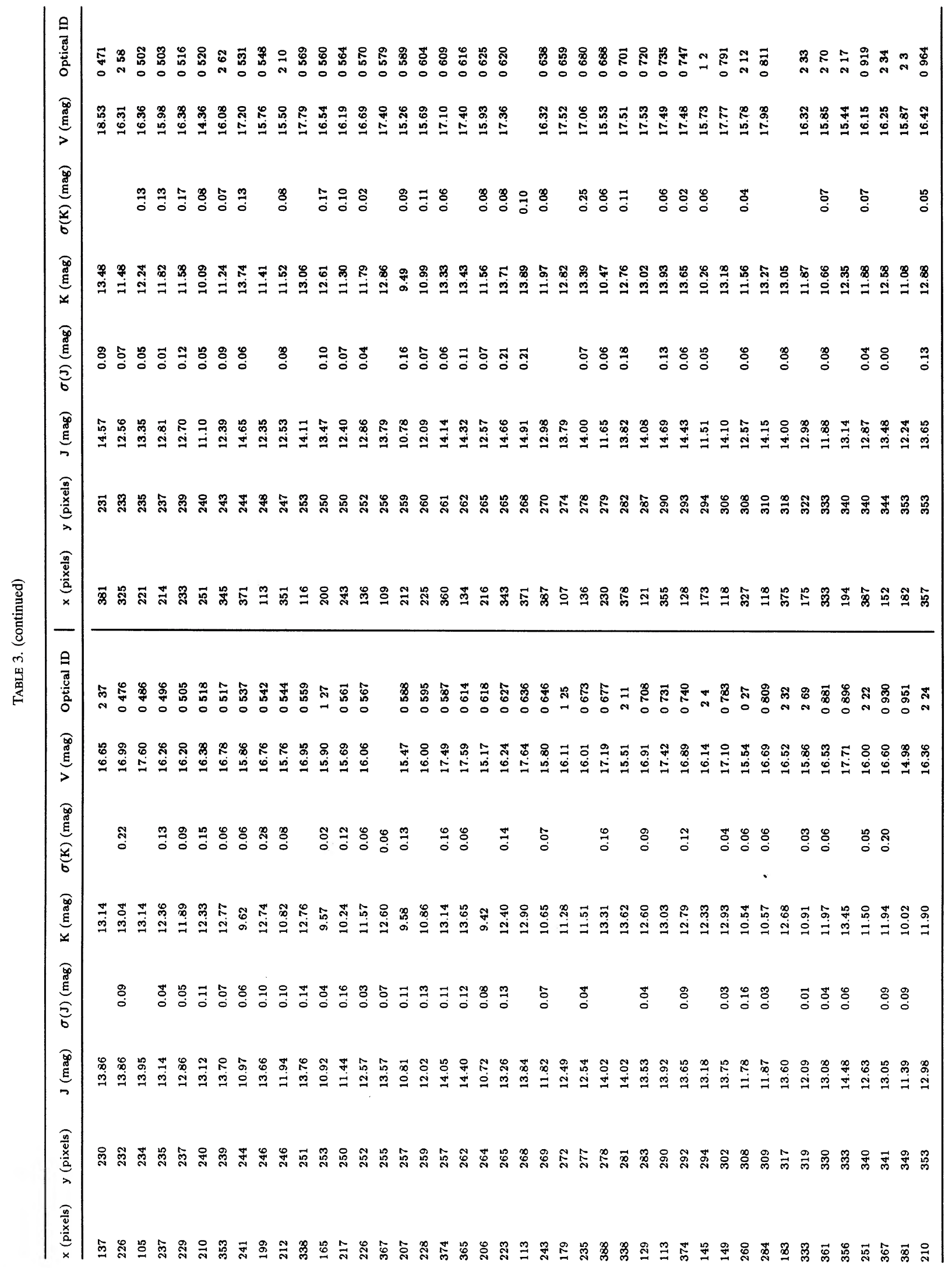




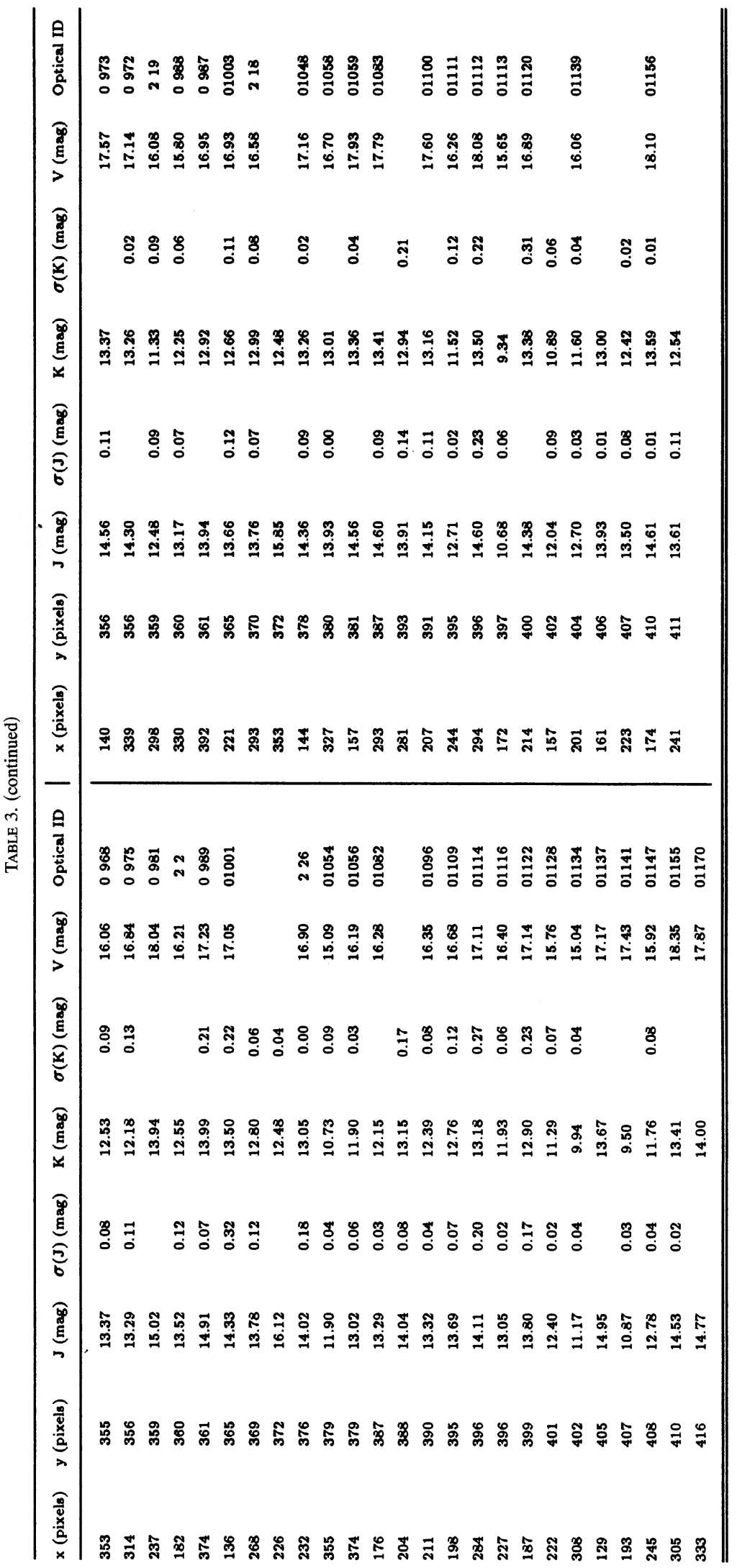



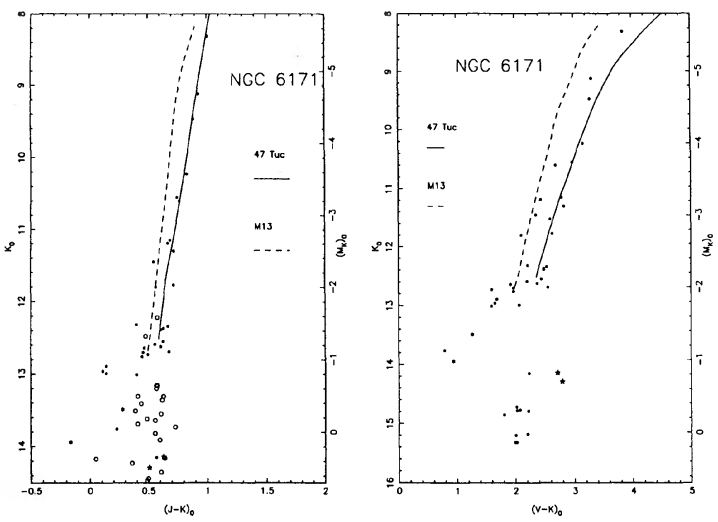

FIG. 4. The same as Figs. 1(a) and 1(b) for NGC 6171. Two definite field stars are plotted with open asterisks, while a filled "*" indicates the single RR Lyrae variable we observed in this cluster.

$B-V$ diagram of DaCosta et al. (1984), and verified by their position in Fig. 4. There are three stars in common with the earlier photometry of Frogel et al. (1983b). They are the three brightest stars observed here. We obtain for them $\Delta(K)$ $=0.03 \mathrm{mag}$ and $\Delta(J-K)=0.05 \mathrm{mag}$ with a dispersion of less than 0.01 mag in both cases. The older measurements are somewhat brighter in both $J$ and $K$.

The presence of RR Lyrae variables offers us a chance to determine the distance modulus in a manner which is much more independent of reddening than the usual optical procedures. We use the infrared period-luminosity relationship established by the work of many people for field RR Lyrae stars and summarized by Jones et al. (1992). Using the periods derived from optical photometry in the catalog of Sawyer Hogg (1973), we determine a distance of $5.2 \pm 0.4 \mathrm{kpc}$. The distance modulus derived from the mean apparent visual magnitude of the horizontal branch which we adopt here is $5.8 \mathrm{kpc}$, in reasonable agreement with that value. Note that the optical distance modulus is critically dependent on the choice of reddening. Additional infrared photometry of RR Lyrae variables in globular clusters should prove very informative.

\subsection{NGC 7099}

NGC 7099 is a low metallicity globular cluster at high galactic latitude with low reddening at a distance of $7.4 \mathrm{kpc}$. We have adopted $\mathrm{E}(B-V)=0.05 \mathrm{mag}$. Optical photometry was given by Dickens (1972), while Alcaino \& Liller (1980) give deeper photographic photometry reaching the main sequence. We have also taken a few $V$ magnitudes from the shortest exposure field studied by Bolte (1987). In addition, to reach the brightest stars, most of which have no published visual photometry, we took two $20 \mathrm{~s}$ exposures with the 1.5 $\mathrm{m}$ telescope at Palomar Mountain, and used the published Dickens photometry to calibrate our DAOPHOT measurements. The major peculiarity of NGC 7099 discerned from these studies is a relative paucity of red giants compared to horizontal branch stars. One speculative explanation advanced by Dickens is that the He abundance in NGC 7099 is

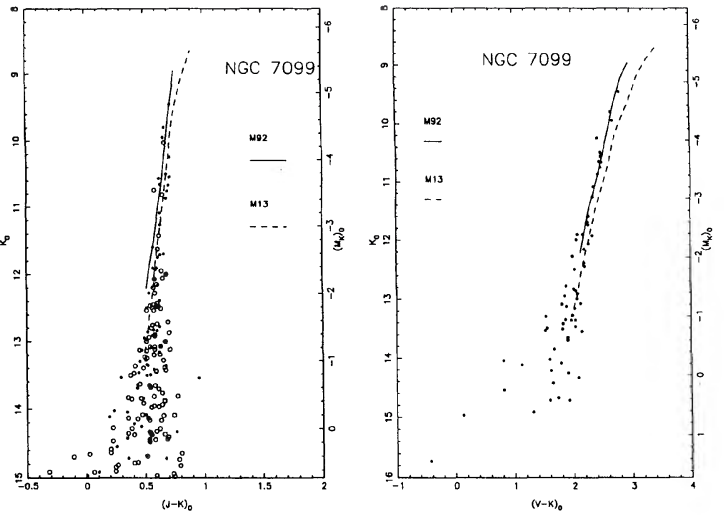

Fig. 5. The same as Figs. 1(a) and 1(b) for NGC 7099. A filled "*” denotes the single RR Lyrae variable we observed in this cluster.

unusually high. We cover three fields on four nights with the infrared array. The first is centered about 1 arcmin north of the cluster center, the second 1 arcmin directly south of the center, and the third is 1 arcmin east of the second field. The scarcity of red giants is apparent from these images. We can resolve stars essentially into the center of the cluster, but the region with a radius of 10 arcsec of the cluster core was eliminated, as the photometry showed a large scatter.

All of the nights on which this cluster was observed from Las Campanas were not photometric. So the images were calibrated by frames taken with the infrared array camera on the $5 \mathrm{~m}$ Hale telescope at Palomar Mountain. This cluster is rather far south to be observed from Palomar with high precision (the airmass always exceeds 1.8 ), and hence there is a $0.04 \mathrm{mag}$ uncertainty in the zero point of the $J-K$ colors. The infrared photometry is given in Table 7; magnitudes given in parenthesis under the column for the optical identification are our new Palomar measurements. Figures 5(a) and 5 (b) present the infrared color-magnitude diagrams with the usual meaning of the symbols.

We use the numbering system of Dickens as it covers the spatial and magnitude range spanned by our data better than does the later work. Note that because of the sparseness of the field and the absence of a sheet of field stars, as well as the better seeing, we are able to reach to $K \approx 16 \mathrm{mag}$, albeit with significant measurement errors. We clearly see the previously known horizontal branch stars in our fields. There are only three RR Lyrae variables known in this cluster (Sawyer Hogg 1973), and one of these falls within the area we studied. For this single variable, imaged at $K$ at a random single phase, and using the infrared period-luminosity law described in the references in Sec. 3.4 on NGC 6171, we obtain a distance modulus within $0.1 \mathrm{mag}$ of that adopted here.

\section{RESULTS}

We discuss two major properties of our data. First we consider the spread in the giant branches of the five globular clusters for various colors, and try to distinguish between the various possible causes of it. We then examine the reddening 


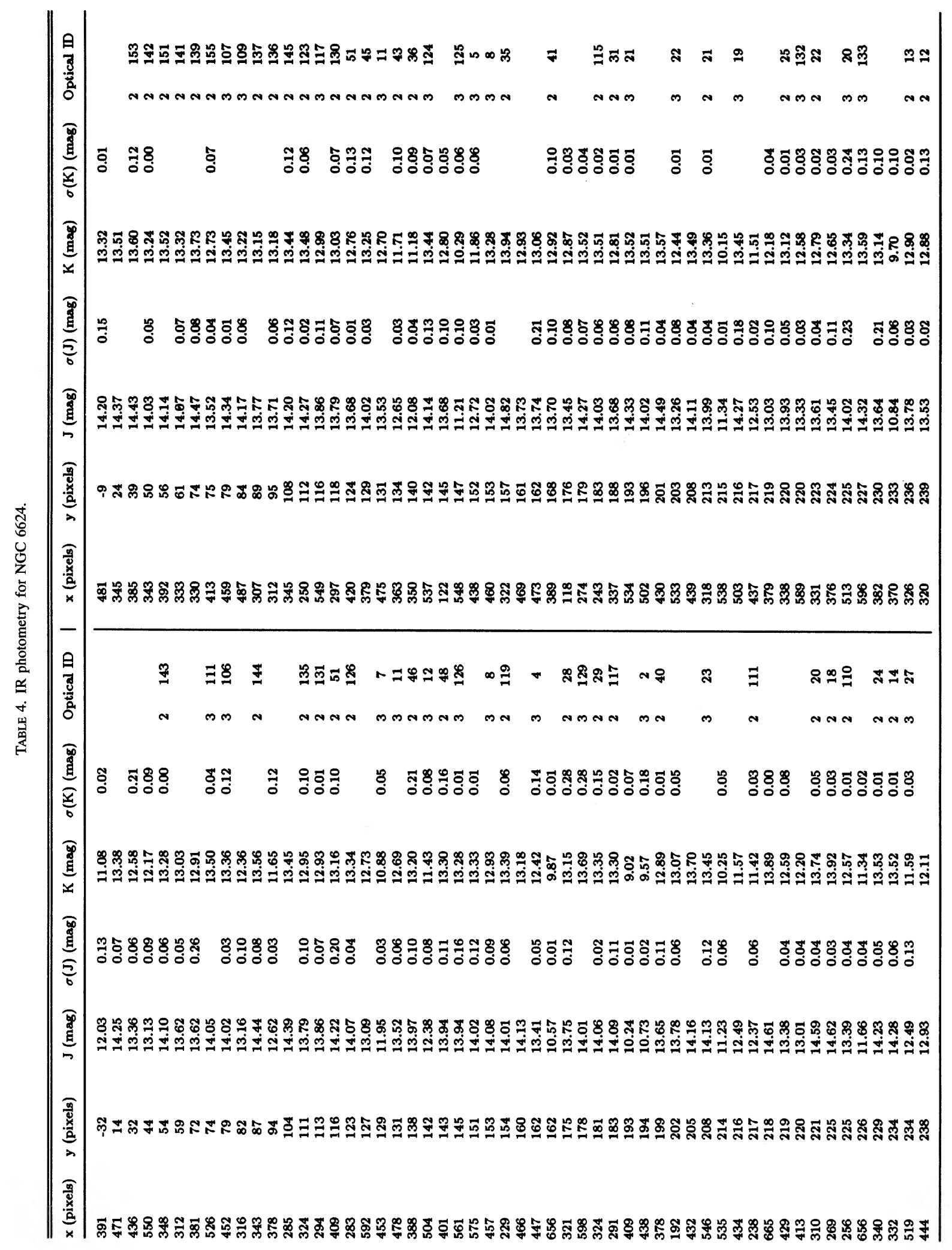




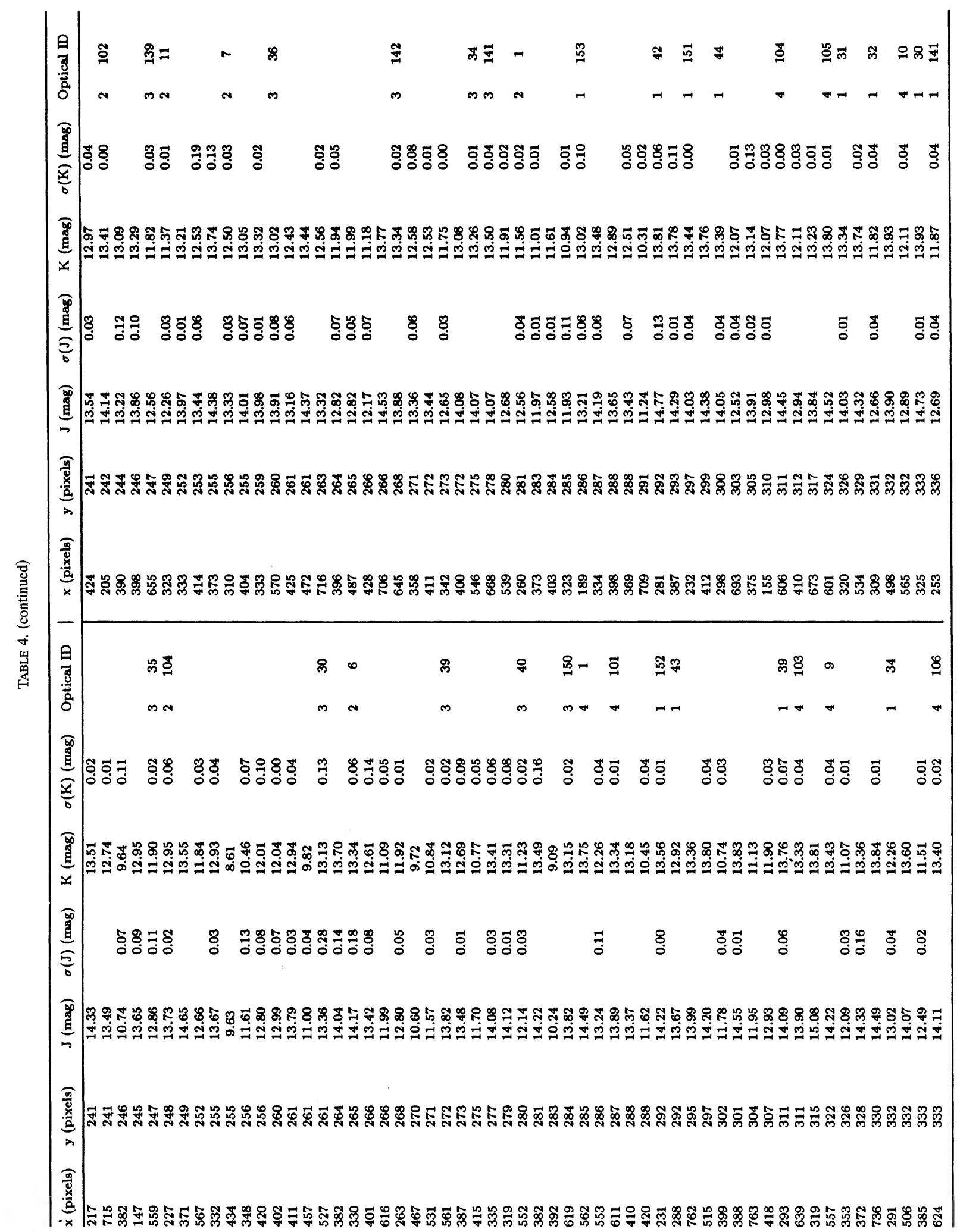


corrected colors of the mean giant branch of each cluster at a fixed luminosity and use those parameters to study the metallicity of this sample of globular clusters.

\subsection{The Widths of the Giant Branches}

An interesting point to note is the relatively large spread in color at a given magnitude seen in the $K,(V-K)$ colormagnitude diagrams. This spread in $V-K$ within a globular cluster along the giant branch is sometimes quite large, reaching $0.6 \mathrm{mag}$ for NGC 6528 . The spread within a given cluster in $J-K$ is smaller, as is the spread in $B-V$. These spreads can arise either from patchy interstellar reddening or from metallicity variations among the stars in a particular globular cluster. The measurements of parameters describing these spreads are given for two luminosities at $K_{0},-3$ and $-4 \mathrm{mag}$ in Table 8 . In general, the color spread measurements are more reliable at the higher luminosity, where the uncertainties in the infrared photometry of any individual star are small and do not contribute. Note that the widths given are full widths of the color distribution, not $\sigma$ values. The values of $\delta(B-V)$ were measured from the colormagnitude diagrams in the references for the optical photometry given in Sec. 3.

We rule out the effect of a strong AGB or of the presence of field stars. Even a strong AGB can only produce a range in colors which is small compared to that under discussion here. Also, the AGB component will appear as a distinct feature blueward of the first ascent red giants, especially at low luminosities, rather than as a complete blurring out of the giant branch in color. Note that the observed spreads are seen all along the giant branch independent of luminosity (until the luminosity is so low that the infrared magnitudes measured with a $1 \mathrm{~m}$ telescope become very imprecise). Some field star contamination of our sample has undoubtedly occurred, and we have ignored all stars bluer than the M13 locus in NGC 5927 and in NGC 6624. Background frames taken 10 arcmin away from the cluster field for each of the five clusters show that it is in general minimal and not important here.

We can use the ratio of width of the giant branch at a fixed $M_{K_{0}}$ in various colors, particularly when the spreads are large, to try to differentiate between the effects of patchy interstellar reddening and those of a real metallicity spread within these metal rich clusters. Recall that there is essentially no data on the possible presence of a metallicity range within the $\mathrm{r}$ iry metal rich galactic globular clusters. Furthermore, spectroscopic studies of sufficient accuracy to detect small abundance variations within a globular cluster are of necessity confined to small samples of stars. Unless very large samples are examined spectroscopically, this issue cannot be probed, as any specific star which shows an abnormality could always be dismissed as a field star.

The dependence of the predicted width of the giant branch in various colors if patchy reddening occurs is deduced from the wavelength dependence of the interstellar reddening law. The dependence of the giant branch location for various infrared colors on metallicity is taken from Eq. (7) of Frogel et al. (1983a). Table 9 compares the ratios of the color 
TABLE 5. $V$ and $R$ photometry for NGC 6624 .

\begin{tabular}{|c|c|c|c|c|c|c|c|c|c|c|c|}
\hline$\underset{\text { (pixels) }}{\mathbf{x}}$ & $\begin{array}{c}\mathbf{y} \\
\text { (pixels) }\end{array}$ & Star ID & $\begin{array}{c}\text { V(CTIO) } \\
\text { (mag) }\end{array}$ & $\begin{array}{c}\Delta(\mathrm{V}) \\
(\mathrm{mag})\end{array}$ & $\begin{array}{l}V-R \\
(\mathrm{mag})\end{array}$ & $\underset{\text { (pixels)( }}{\mathbf{x}}$ & $\underset{\text { ixels) }}{y}$ & Star ID & $\begin{array}{c}\text { V(CTIO) } \\
\text { (mag) }\end{array}$ & $\begin{array}{c}\Delta(\mathrm{V}) \\
(\mathrm{mag})\end{array}$ & $\begin{array}{l}\text { V-R } \\
\text { (mag) }\end{array}$ \\
\hline 391 & -32 & & 14.77 & & 0.89 & 481 & -9 & & 16.48 & & 0.69 \\
\hline 471 & 14 & & 16.43 & & 0.68 & 345 & 24 & & 16.51 & & 0.70 \\
\hline 436 & 32 & & 15.51 & & 0.73 & 385 & 39 & $2-153$ & 16.61 & 0.06 & 0.73 \\
\hline 550 & 44 & & 15.75 & & 0.82 & 343 & 50 & $2-142$ & 16.12 & 0.07 & 0.66 \\
\hline 348 & 54 & 2- 143 & 16.45 & 0.13 & 0.72 & 392 & 56 & 2- 151 & 16.14 & 0.10 & 0.71 \\
\hline 312 & 59 & & 16.06 & & $0: 75$ & 333 & 61 & $2-141$ & 16.18 & 0.07 & 0.66 \\
\hline 381 & 72 & & 15.06 & & 0.55 & 330 & 74 & 2- 139 & 16.61 & 0.12 & 0.70 \\
\hline 526 & 74 & 3- 111 & 16.19 & 0.09 & 0.64 & 413 & 75 & 2- 155 & 16.14 & 0.08 & 0.86 \\
\hline 452 & 79 & 3- 106 & 16.10 & 0.05 & 0.60 & 459 & 79 & 3- 107 & 16.61 & 0.09 & 0.71 \\
\hline 316 & 82 & & 15.81 & & 0.83 & 487 & 84 & 3- 109 & 16.10 & 0.06 & 0.62 \\
\hline 343 & 87 & $2-144$ & 16.75 & 0.09 & 0.70 & 378 & 94 & & 15.07 & & 0.75 \\
\hline 345 & 108 & $2-145$ & 16.36 & 0.09 & 0.68 & 324 & 111 & 2- 135 & 16.43 & 0.11 & 0.84 \\
\hline 250 & 112 & $2-123$ & 16.65 & 0.10 & 0.81 & 294 & 113 & $2-131$ & 16.18 & 0.12 & 0.80 \\
\hline 549 & 116 & 3- 117 & 15.90 & 0.12 & 0.63 & 409 & 116 & 2- 51 & 16.73 & -0.64 & 0.76 \\
\hline 297 & 118 & $2-130$ & 15.93 & 0.16 & 0.68 & 283 & 123 & 2- 126 & 16.21 & 0.10 & 0.67 \\
\hline 420 & 124 & 2- 51 & 16.02 & 0.07 & 0.75 & 379 & 129 & $2-45$ & 16.02 & 0.14 & 0.62 \\
\hline 453 & 129 & 3- 7 & 14.73 & -0.01 & 0.87 & 475 & 131 & 3- 11 & 16.05 & 0.13 & 0.80 \\
\hline 478 & 131 & 3- 11 & 16.05 & 0.13 & 0.80 & 363 & 134 & 2- 43 & 15.21 & 0.01 & 0.82 \\
\hline 388 & 138 & $2-46$ & 16.06 & 0.09 & 0.65 & 350 & 140 & $2-36$ & 14.70 & 0.01 & 0.83 \\
\hline 504 & 142 & 3- 12 & 14.91 & -0.05 & 0.77 & 537 & 142 & 3- 124 & 16.45 & 0.10 & 0.66 \\
\hline 401 & 143 & 2- 48 & 16.06 & 0.14 & 0.61 & 122 & 145 & & 16.61 & & 0.92 \\
\hline 561 & 145 & $3-126$ & 16.03 & 0.07 & 0.59 & 548 & 147 & 3- 125 & 13.77 & 0.03 & 0.78 \\
\hline 575 & 151 & & 16.25 & & 0.63 & 438 & 152 & 3- 5 & 15.10 & -0.06 & 0.73 \\
\hline 457 & 153 & 3- 8 & 16.27 & -0.03 & 0.71 & 460 & 153 & $3-8$ & 16.27 & -0.03 & 0.71 \\
\hline 229 & 154 & 2- 119 & 16.14 & 0.15 & 0.66 & 466 & 160 & & 16.12 & & 0.65 \\
\hline 447 & 162 & 3- 4 & 15.74 & 0.07 & 0.72 & 656 & 162 & & 12.98 & & 0.71 \\
\hline 389 & 168 & 2- 41 & 15.72 & 0.06 & 0.64 & 321 & 175 & 2- 28 & 15.84 & 0.09 & 0.68 \\
\hline 118 & 176 & & 15.59 & & 0.63 & 598 & 178 & 3- 129 & 16.01 & 0.10 & 0.59 \\
\hline 274 & 179 & & 16.51 & & 0.66 & 324 & 181 & 2- 29 & 16.29 & 0.10 & 0.69 \\
\hline 243 & 183 & 2- 115 & 15.76 & 0.10 & 0.56 & 291 & 183 & $2-117$ & 16.01 & 0.13 & 0.61 \\
\hline 337 & 188 & 2- 31 & 15.92 & 0.12 & 0.72 & 409 & 193 & & 14.09 & & 1.12 \\
\hline 534 & 193 & 3- 21 & 16.50 & 0.09 & 0.66 & 438 & 194 & 3- 2 & 13.93 & 0.05 & 0.99 \\
\hline 502 & 196 & & 15.98 & & 0.58 & 378 & 199 & $2-40$ & 15.65 & 0.05 & 0.66 \\
\hline 430 & 201 & & 16.45 & & 0.68 & 192 & 202 & & 15.98 & & 0.72 \\
\hline 533 & 203 & 3- 22 & 15.69 & 0.12 & 0.71 & 432 & 205 & & 16.38 & & 0.67 \\
\hline 439 & 208 & & 15.98 & & 0.60 & 546 & 208 & 3- 23 & 16.59 & 0.06 & 0.66 \\
\hline 318 & 213 & 2- 21 & 15.92 & 0.08 & 0.61 & 535 & 214 & & 14.26 & & 0.91 \\
\hline 538 & 215 & & 14.26 & & 0.91 & 434 & 216 & & 14.99 & & 0.77 \\
\hline 503 & 216 & 3- 19 & 16.01 & 0.08 & 0.59 & 238 & 217 & 2- 111 & 14.93 & -0.03 & 0.83 \\
\hline 437 & 217 & & 14.99 & & 0.77 & 665 & 218 & & 16.69 & & 0.62 \\
\hline 379 & 219 & & 15.34 & & 0.73 & 429 & 219 & & 15.74 & & 0.73 \\
\hline 338 & 220 & 2- 25 & 16.01 & 0.08 & 0.67 & 413 & 220 & & 15.21 & & 0.69 \\
\hline 589 & 220 & 3- 132 & 15.77 & 0.12 & 0.69 & 310 & 221 & $2-20$ & 16.67 & 0.02 & 0.70 \\
\hline 331 & 223 & $2-22$ & 15.84 & 0.12 & 0.70 & 269 & 225 & 2- 18 & 16.78 & 0.00 & 0.68 \\
\hline 376 & 224 & & 15.59 & & 0.70 & 256 & 225 & 2- 110 & 15.79 & 0.11 & 0.75 \\
\hline 513 & 225 & $3-20$ & 15.96 & 0.07 & 0.61 & 656 & 226 & & 12.94 & & 0.36 \\
\hline 596 & 227 & 3- 133 & 16.56 & 0.11 & 0.64 & 340 & 229 & $2-24$ & 15.94 & 0.03 & 0.58 \\
\hline 382 & 230 & & 15.48 & & 0.59 & 332 & 234 & 2- 14 & 16.51 & 0.05 & 0.69 \\
\hline 370 & 233 & & 13.97 & & 0.99 & 519 & 234 & 3- 27 & 15.09 & -0.08 & 0.77 \\
\hline 326 & 236 & $2-13$ & 16.27 & 0.11 & 0.74 & 444 & 238 & & 15.28 & & 0.86 \\
\hline 320 & 239 & 2- 12 & 15.74 & 0.09 & 0.68 & 217 & 241 & & 16.55 & & 0.71 \\
\hline 715 & 241 & & 16.00 & & 0.73 & 205 & 242 & 2- 102 & 16.03 & 0.14 & 0.58 \\
\hline 382 & 246 & & 13.93 & & 0.99 & 390 & 244 & & 14.81 & & 0.50 \\
\hline 147 & 245 & & 16.45 & & 0.86 & 559 & 247 & 3- 35 & 15.57 & 0.12 & 0.82 \\
\hline 655 & 247 & 3- 139 & 15.01 & 0.00 & 0.69 & 227 & 248 & 2- 104 & 16.01 & 0.12 & 0.71 \\
\hline 323 & 249 & 2- 11 & 14.80 & -0.07 & 0.78 & 371 & 249 & & 16.35 & & 0.65 \\
\hline 333 & 252 & & 15.95 & & 0.61 & 567 & 252 & & 15.10 & & 0.72 \\
\hline 332 & 255 & & 15.91 & & 0.63 & 373 & 255 & & 15.98 & & 0.58 \\
\hline 434 & 255 & & 13.47 & & 1.18 & 310 & 256 & 2- 7 & 15.67 & 0.09 & 0.73 \\
\hline 348 & 256 & & 14.40 & & 0.92 & 404 & 255 & & 15.66 & & 0.51 \\
\hline 420 & 256 & & 14.77 & & 0.67 & 333 & 259 & & 15.94 & & 0.60 \\
\hline 402 & 260 & & 15.06 & & 0.65 & 570 & 260 & $3-36$ & 16.49 & 0.08 & 0.80 \\
\hline 411 & 261 & & 15.77 & & 0.67 & 425 & 261 & & 15.30 & & 0.60 \\
\hline 457 & 261 & & 14.00 & & 0.94 & 472 & 261 & & 15.79 & & 0.59 \\
\hline 527 & 261 & $3-30$ & 15.86 & 0.07 & 0.62 & 716 & 263 & & 15.76 & & 0.69 \\
\hline 382 & 264 & & 15.97 & & 0.59 & 396 & 264 & & 15.15 & & 0.72 \\
\hline 330 & 265 & $2-6$ & 15.96 & 0.02 & 0.62 & 487 & 265 & & 15.17 & & 0.74 \\
\hline 401 & 266 & & 15.32 & & 0.62 & 428 & 266 & & 14.66 & & 0.77 \\
\hline 616 & 266 & & 15.26 & & 0.96 & 706 & 266 & & 16.89 & & 0.74 \\
\hline 263 & 268 & & 15.39 & & 0.79 & 645 & 268 & 3- 142 & 16.01 & 0.06 & 0.58 \\
\hline 467 & 270 & & 13.91 & & 1.06 & 358 & 271 & & 15.30 & & 0.66 \\
\hline 531 & 271 & & 14.03 & & 0.74 & 561 & 272 & 3- 39 & 15.97 & 0.11 & 0.61 \\
\hline 342 & 273 & & 15.07 & & 0.72 & 387 & 273 & & 15.65 & & 0.64 \\
\hline 400 & 272 & & 15.95 & & 0.59 & 546 & 275 & $3-34$ & 16.43 & 0.10 & 0.69 \\
\hline
\end{tabular}


TABLE 5. (continued)

\begin{tabular}{|c|c|c|c|c|c|c|c|c|c|c|c|}
\hline$\underset{\text { (pixels) }}{\mathbf{x}}$ & $\underset{\text { (pixels) }}{y}$ & Star ID & $\begin{array}{c}\mathrm{V} \text { (CTIO) } \\
\text { (mag) }\end{array}$ & $\begin{array}{l}\Delta(\mathrm{V}) \\
\text { (mag) }\end{array}$ & $\begin{array}{l}V-R \\
(\mathrm{mag})\end{array}$ & $\begin{array}{c}\mathbf{x} \\
\text { (pixels) }\end{array}$ & $\underset{\text { pixels) }}{\mathbf{y}}$ & Star ID & $\begin{array}{c}\mathrm{V}(\mathrm{CTIO}) \\
\text { (mag) }\end{array}$ & $\begin{array}{l}\Delta(\mathrm{V}) \\
(\mathrm{mag})\end{array}$ & $\begin{array}{l}V-R \\
\text { (mag) }\end{array}$ \\
\hline 400 & 272 & & 15.95 & & 0.59 & 546 & 275 & $3-34$ & 16.43 & 0.10 & 0.69 \\
\hline 668 & 278 & 3- 141 & 16.14 & 0.08 & 0.58 & 319 & 279 & & 16.31 & & 0.69 \\
\hline 539 & 280 & & 15.09 & & 0.69 & 552 & 280 & $3-40$ & 14.90 & & 0.80 \\
\hline 260 & 281 & 2- 1 & 15.74 & 0.16 & 0.98 & 382 & 281 & & 15.82 & & 0.57 \\
\hline 373 & 283 & & 14.44 & & 0.76 & 392 & 283 & & 13.73 & & 1.08 \\
\hline 403 & 284 & & 15.06 & & 0.75 & 619 & 284 & 3- 150 & 15.91 & 0.09 & 0.59 \\
\hline 323 & 285 & & 14.48 & & 0.79 & 562 & 285 & 4- 1 & 16.82 & 0.07 & 0.71 \\
\hline 189 & 286 & $1-153$ & 15.53 & 0.10 & 0.74 & 553 & 286 & & 16.01 & & 0.83 \\
\hline 334 & 287 & & 15.99 & & 0.61 & 611 & 287. & 4- 101 & 15.49 & 0.09 & 0.50 \\
\hline 398 & 288 & & 15.90 & & 0.63 & 410 & 288 & & 15.45 & & 0.63 \\
\hline 369 & 288 & & 15.56 & & 0.74 & 420 & 288 & & 13.81 & & 0.65 \\
\hline 709 & 291 & & 14.24 & & 0.86 & 231 & 292 & 1- 152 & 16.50 & 0.07 & 0.68 \\
\hline 281 & 292 & 1. 42 & 16.87 & 0.12 & 0.70 & 288 & 292 & $1-43$ & 15.77 & 0.09 & 0.64 \\
\hline 387 & 293 & & 16.10 & & 0.58 & 762 & 295 & & 16.07 & & 0.57 \\
\hline 232 & 297 & $1-151$ & 16.03 & 0.21 & 0.61 & 515 & 297 & & 16.34 & & 0.61 \\
\hline 412 & 299 & & 15.81 & & 0.58 & 399 & 302 & & 14.40 & & 0.82 \\
\hline 298 & 300 & $1-44$ & 15.95 & 0.09 & 0.60 & 388 & 301 & & 15.99 & & 0.65 \\
\hline 693 & 303 & & 14.15 & & 0.49 & 763 & 304 & & 14.49 & & 0.73 \\
\hline 375 & 305 & & 15.78 & & 0.60 & 418 & 307 & & 14.90 & & 0.70 \\
\hline 155 & 310 & & 15.68 & & 0.80 & 293 & 311 & 1- 39 & 15.34 & -0.02 & 0.44 \\
\hline 606 & 311 & 4- 104 & 16.90 & 0.06 & 0.75 & 639 & 311 & 4- 103 & 15.94 & 0.07 & 0.57 \\
\hline 410 & 312 & & 15.00 & & 0.68 & 319 & 315 & & 16.70 & & 0.64 \\
\hline 673 & 317 & & 16.00 & & 0.58 & 557 & 322 & 4- 9 & 16.51 & 0.07 & 0.69 \\
\hline 601 & 324 & 4- 105 & 16.87 & 0.07 & 0.71 & 353 & 326 & & 14.70 & & 0.81 \\
\hline 320 & 326 & $1-31$ & 15.96 & 0.10 & 0.62 & 372 & 328 & & 16.22 & & 0.65 \\
\hline 534 & 329 & & 16.52 & & 0.63 & 736 & 330 & & 16.46 & & 0.64 \\
\hline 309 & 331 & & 15.17 & & 0.78 & 291 & 332 & $1-34$ & 15.28 & 0.01 & 0.69 \\
\hline 498 & 332 & & 15.97 & & 0.64 & 506 & 332 & & 16.02 & & 0.57 \\
\hline 565 & 332 & 4- 10 & 15.43 & 0.09 & 0.73 & 385 & 333 & & 14.96 & & 0.78 \\
\hline 325 & 333 & 1- 30 & 16.74 & 0.07 & 0.62 & 624 & 333 & 4- 106 & 16.57 & 0.08 & 0.73 \\
\hline 253 & 336 & $1-141$ & 15.07 & -0.01 & 0.73 & 670 & 336 & & 15.97 & & 0.67 \\
\hline 299 & 337 & 1- 33 & 14.90 & -0.04 & 0.80 & 587 & 342 & 4- 112 & 15.17 & 0.02 & 0.76 \\
\hline 762 & 343 & & 16.09 & & 0.55 & 406 & 349 & & 14.47 & & 0.87 \\
\hline 555 & 350 & 4- 12 & 15.60 & 0.07 & 0.61 & 395 & 355 & & 15.73 & & 0.83 \\
\hline 385 & 364 & & 15.59 & & 0.60 & 542 & 366 & 4- 21 & 14.62 & -0.03 & 0.82 \\
\hline 532 & 367 & $4-20$ & 16.15 & 0.15 & 0.66 & 574 & 367 & 4- 119 & 16.59 & 0.14 & 0.60 \\
\hline 378 & 368 & & 15.53 & & 0.74 & 129 & 369 & & 15.53 & & 0.51 \\
\hline 570 & 370 & $4-120$ & 15.59 & & 0.71 & 299 & 376 & $1-20$ & 16.69 & 0.02 & 0.69 \\
\hline 235 & 378 & $1-137$ & 16.89 & 0.07 & 0.74 & 566 & 378 & 4- 121 & 16.81 & 0.06 & 0.72 \\
\hline 635 & 378 & 4- 111 & 15.43 & 0.01 & 0.61 & 215 & 381 & $1-135$ & 16.39 & 0.10 & 0.80 \\
\hline 689 & 381 & & 16.07 & & 0.56 & 668 & 383 & & 15.94 & & 0.72 \\
\hline 655 & 387 & & 16.60 & & 0.67 & 353 & 388 & & 15.04 & & 0.81 \\
\hline 387 & 390 & & 15.94 & & 0.58 & 588 & 397 & & 15.90 & & 0.61 \\
\hline 736 & 399 & & 16.43 & & 0.62 & 561 & 406 & & 15.04 & & 0.69 \\
\hline 755 & 416 & & 16.24 & & 0.57 & 629 & 417 & & 16.53 & & 0.65 \\
\hline 723 & 420 & & 16.06 & & 0.76 & & & & & & \\
\hline
\end{tabular}

widths (using the average of the values at the two luminosities $K_{0}=-3$ and -4 mag given in Table 8) measured in the sample globular clusters (omitting NGC 7099, as the reddening is too low) in $(V-K),(J-K)$, and $(B-V)$ with those expected if the entire effect is caused by patchy interstellar reddening or if it is caused exclusively by abundance variations. The allowed ranges for the color width ratios given in Table 9 are obtained from the uncertainties for the color widths given in Table 8 . The last column of Table 9 gives the range in $\mathrm{E}(B-V), \delta[\mathrm{E}(B-V)]$, as inferred from $\delta(V-K)$, divided by the mean reddening for the cluster.

In all cases and for all colors from $B$ to $K$ (with the single exception discussed below) the ratios of the color widths of the globular cluster giant branches are completely consistent with all the spreads arising from patchy interstellar reddening. No cluster behaves as though metallicity variations dominate the cause of the color spreads.

The $(J-K)$ data are least useful here. The reddening is small across that bandpass, and hence, if the widths are due to patchy interstellar reddening, they too will be small in the $K,(J-K)$ diagram. This is in fact what is observed (see Table 8). The only case which slightly violates the perfect agreement of interstellar reddening with the observed color spread ratios is that of $\delta(V-K) / \delta(J-K)$ in NGC 6624. Perhaps the uncertainties in the color widths have been underestimated, or the effect of field stars and/or AGB stars is becoming important given that the color widths, particularly $\delta(J-K)$, are now small.

To show exactly how much one can constrain $\delta[\mathrm{Fe} / \mathrm{H}]$ using the ratios of the color widths, Fig. 6 illustrates the situation for NGC 6528, the cluster with the largest reddening. The regime allowed by the observed color widths $[\delta(V-K)=0.64 \pm 0.05 \mathrm{mag}$ and $\delta(B-V)=0.23 \pm 0.04 \mathrm{mag}]$ is shown by solid lines for $(B-V)$ and dashed lines for $(V-K)$. The intersection of the two families of curves is the allowed region. The measured values in Table 8 agree with the predictions for no metallicity variations whatsoever, while at most $\delta[\mathrm{Fe} / \mathrm{H}]=0.35$ dex is allowed by the uncertain- 


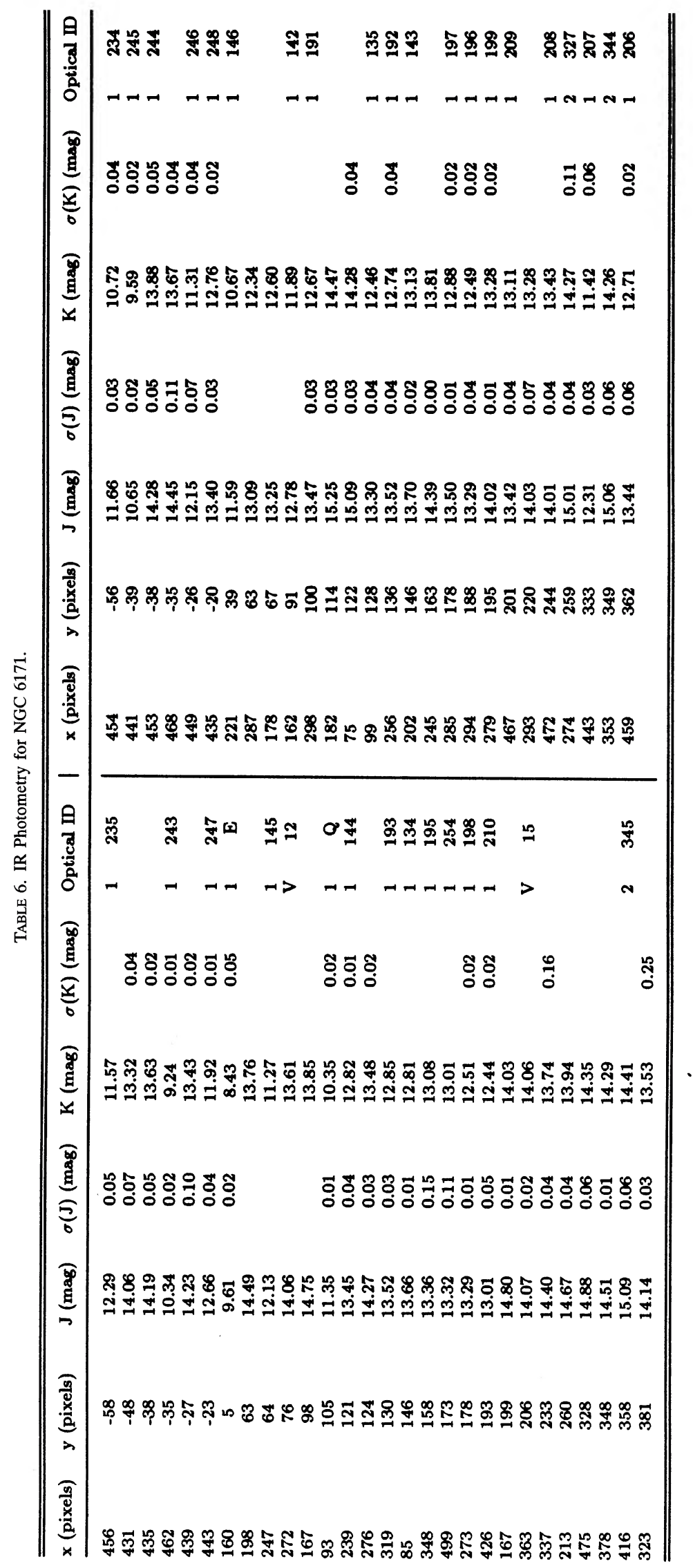




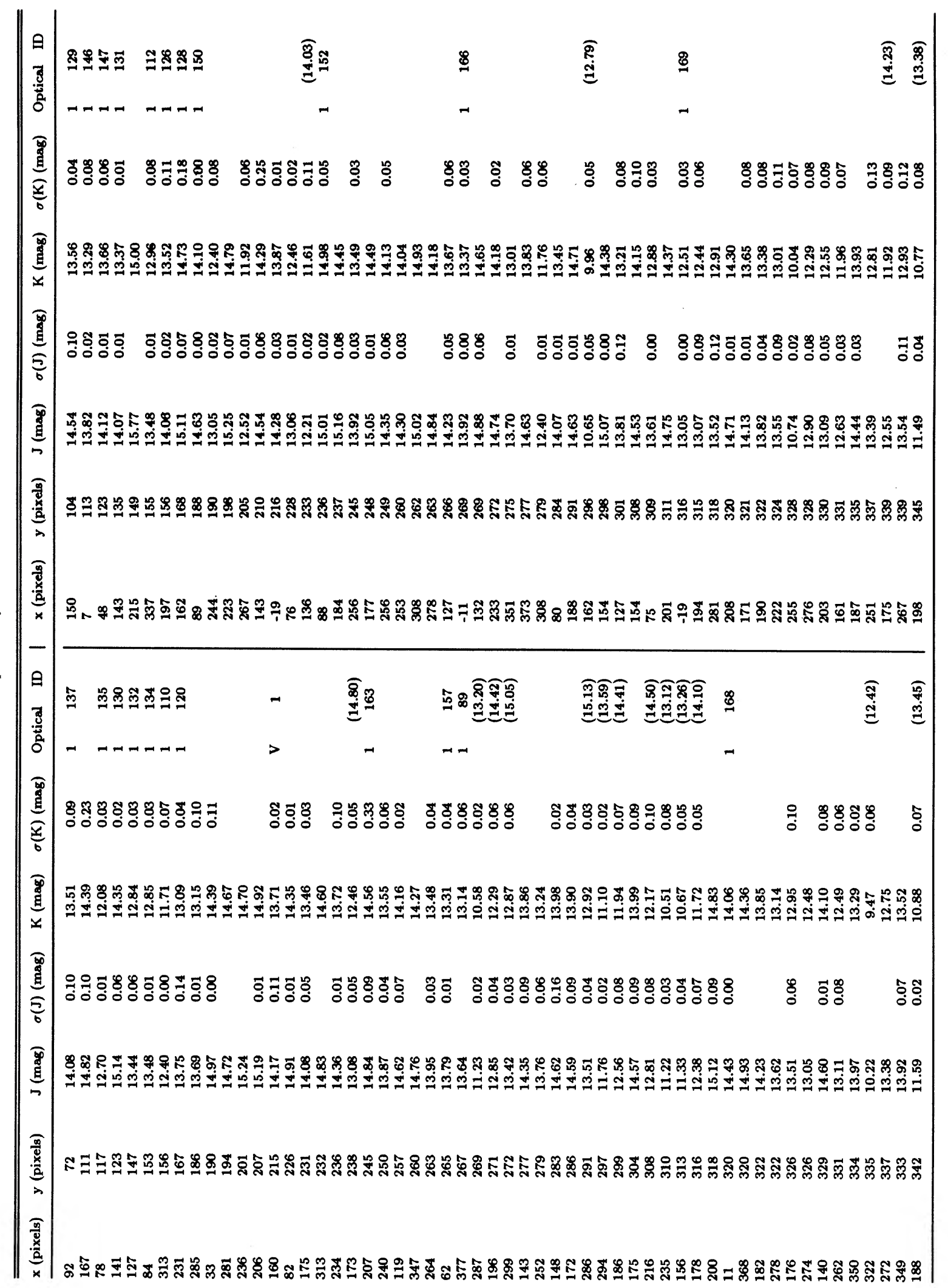




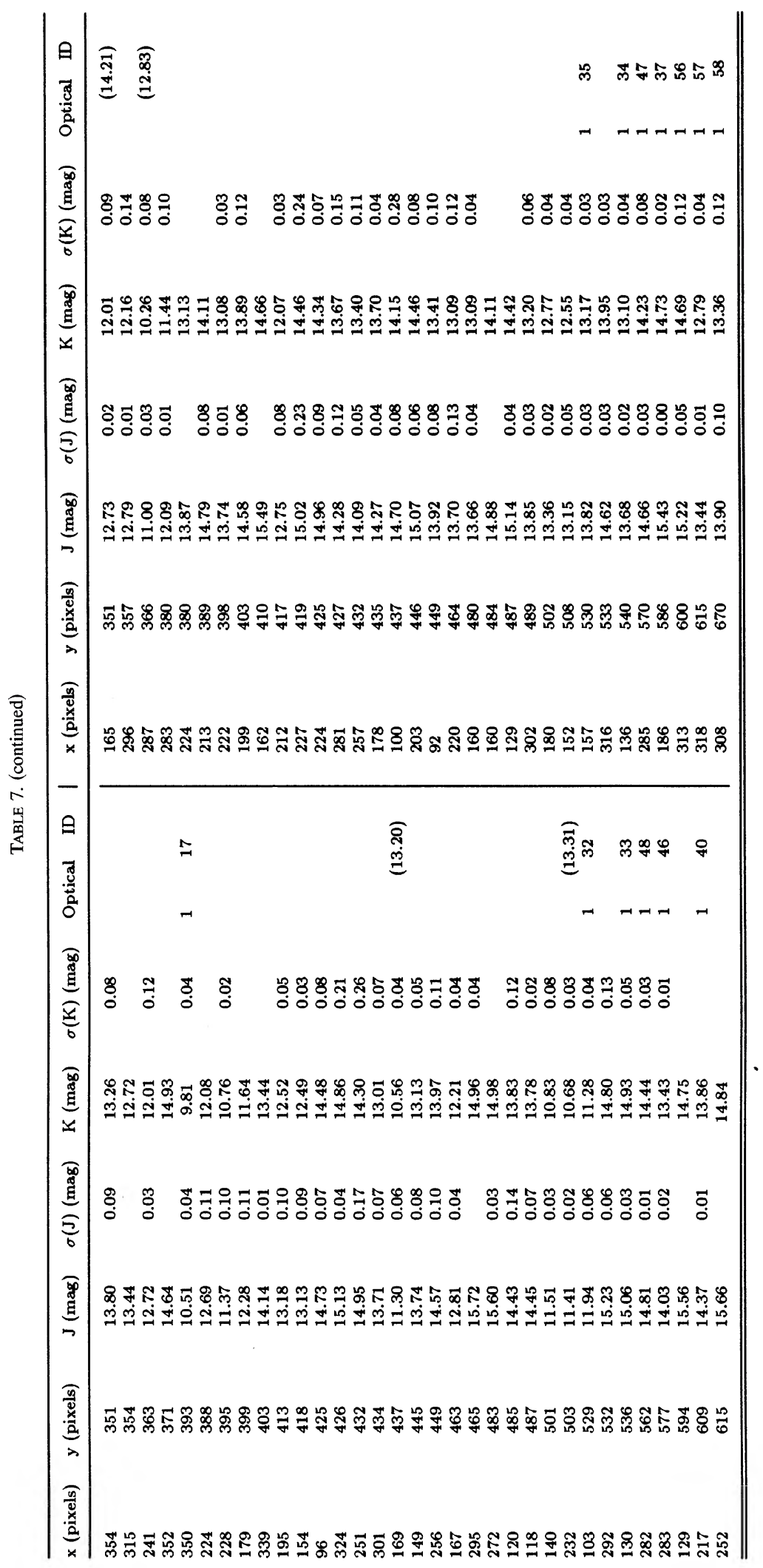


ties in the color widths. If the uncertainty in $\delta(B-V)$ could be reduced, the upper limit on $\delta[\mathrm{Fe} / \mathrm{H}]$ could be made much lower.

For clusters with low interstellar reddening, a small upper limit to the range in $V-K$ along the giant branch is a very effective way to constrain internal metallicity variations. Equation (7) of Frogel et al. (1983a) shows that if $\delta(V-K)$ $<0.10 \mathrm{mag}$, an easily measured value in the absence of differential reddening, then $\delta[\mathrm{Fe} / \mathrm{H}]<0.07 \mathrm{dex}$. This is the case for NGC 7099.

Note that $\delta(B-V)$ as deduced from $\delta(V-K)$ assuming only patchy interstellar reddening is contributing [which number is always close to the measured $\delta(B-V)$ value] implies a variation in the reddening (measured in magnitudes) of $30 \%$ to $40 \%$ across small fields within a globular cluster. Given the modest reddening (0.3-0.6 mag) for these objects, that is rather surprising. The ISM is very patchy on very small spatial scales.

\subsection{The Colors of the Giant Branches}

Frogel et al. (1983a) demonstrated that the color of the giant branch of a globular cluster in the infrared depends primarily on the metallicity of the cluster. Although in a theoretical $\log \left(T_{\text {eff }}\right)-M_{\text {bol }}$ plane, the offset with metallicity is roughly independent of height along the giant branch (Green et al. 1987), this translates in the observational plane into increasingly larger changes in color as the giant branch gets cooler near the tip. Thus, we use the measurements of color of the giant branch in $(J-K)_{0}$ and $(V-K)_{0}$ made at $M_{K_{0}}=-5$ mag. Table 10 gives measurements of the mean color of the giant branch in the five sample globular clusters at $M_{K_{0}}=-5$ and $-4 \mathrm{mag}$, together with the adopted reddening and distance moduli.

Colors for the giant branches of three calibrating clusters, 47 Tuc, M13, and M92, are also given, based on the data in Cohen et al. (1978) or in Frogel et al. (1981). The ridge lines in $(V-K)_{0}$ are in fact tabulated in the last reference. We have corrected the 47 Tuc ridge lines for the different assumption regarding the absolute magnitude of the horizontal branch made here as compared to that adopted by Frogel et al. (1981).

To our sample, we add the very metal rich galactic globular cluster NGC 6553, with infrared photometry of the upper giant branch by Davidge \& Simons (1994) and visual photometry from Hartwick (1975).

We use a weighting of 3 for abundance from $(V-K)_{0}$ as compared to that from $(J-K)_{0}$ because of the much lower sensitivity of the mean $(J-K)$ color of a cluster giant branch at a fixed $M_{K_{0}}$ to abundance changes. Furthermore, we must extrapolate to higher metallicities than 47 Tuc even though we lack any calibrating clusters more metal rich than 47 Tuc. Since the $(J-K)$ gradients are relatively small, we used the gradients in color between M13 and 47 Tuc at $M_{K_{0}}=-5$ to extrapolate to metallicities higher than that of 47 Tuc, assumed to be at $[\mathrm{Fe} / \mathrm{H}]=-0.71 \mathrm{dex}$. When applied to $(V-K)$, this leads to problems, in that the giant branches, particularly near their tips, become acceleratingly redder as the metallicity increases in a nonlinear way, and we would deduce abun-

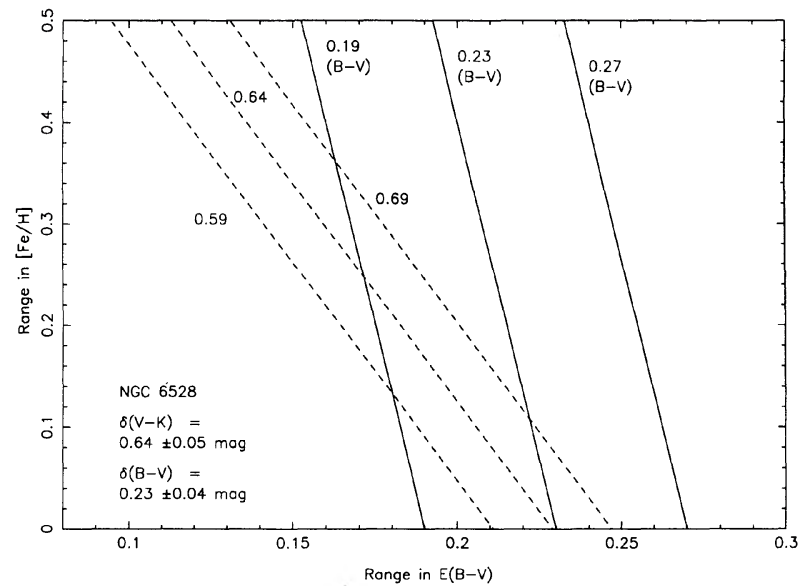

FIG. 6. The permitted regime of $B-V$ or $V-K$ color and range in $[\mathrm{Fe} / \mathrm{H}]$ that match the observed $\delta(B-V)$ or $\delta(V-K)$ color spreads in NGC 6528 is shown. The intersection of the two families of curves is the permitted region.

dances which significantly exceed solar metallicity for the most metal rich clusters studied here. To minimize this effect, we use NGC 6171, whose metallicity can be well established through the procedure described above, as its $[\mathrm{Fe} / \mathrm{H}]$ lies between that of $47 \mathrm{Tuc}$ and that of M13. For the globular clusters more metal rich than 47 Tuc, we then use the gradients determined by comparing $(V-K)_{0}$ at a fixed $M_{K_{0}}$ between the NGC 6171 giant branch and that of 47 Tuc. That gradient $\Delta[\mathrm{Fe} / \mathrm{H}] / \Delta(V-K)_{0}$ is about $30 \%$ smaller than if the 47 Tuc giant branch were compared to that of M13. We do not know how much the gradient continues to decrease as $[\mathrm{Fe} / \mathrm{H}]$ increases still further.

Several attempts have been made to determine the metallicity of these very metal rich clusters. Two of these clusters were included in Frogel et al.'s (1983a) analysis of infrared photometry for the brightest part of the giant branch, two are included in Cohen's (1983) low-dispersion spectroscopic analysis of individual stars, all are included in Zinn's (1980) study of Ca II absorption in the integrated light, and three are included in Armandroff \& Zinn's (1988) analysis of Ca II absorption in the infrared triplet in the integrated light.

Table 11 summarizes the situation. The abundances derived by various authors have been linearly shifted in an appropriate way to force the same abundance for 47 Tuc, -0.71 dex. Typical errors cited for each determination are $\pm 0.15 \mathrm{dex}$, but this does not include any calibration or scale error. While the lower end is fixed by 47 Tuc, which has been extensively studied by many people, the extension in abundance above 47 Tuc is poorly calibrated in all of these analyses.

Given the magnitude of the uncertainties in all the abundance determinations, and considering that none of the schemes is well calibrated for objects more metal-rich than 47 Tuc, the results are encouraging. All the schemes rank the clusters in metallicity in the same order. However, we have a small problem (not more than 0.4 dex in total) with the details of the abundance values for the three metal rich clusters, in that compared to the prior analyses. NGC 6528 and NGC 
TABLE 8. Observed width of the giant branch for five globular clusters.

\begin{tabular}{|c|c|c|c|c|c|c|c|}
\hline Cluster & $\begin{array}{c}\mathrm{E}(B-V) \\
(\mathrm{mag})\end{array}$ & $\begin{array}{c}\delta(B-V) \\
\left(M_{K_{0}}=-3\right)\end{array}$ & $\begin{array}{c}\delta(B-V) \\
\left(M_{K_{0}}=-4\right)\end{array}$ & $\begin{array}{c}\delta(J-K) \\
\left(M_{K_{0}}=-3\right)\end{array}$ & $\begin{array}{c}\delta(J-K) \\
\left(M_{K_{0}}=-4\right)\end{array}$ & $\begin{array}{c}\delta(V-K) \\
\left(M_{K_{0}}=-3\right)\end{array}$ & $\begin{array}{c}\delta(V-K) \\
\left(M_{K_{0}}=-4\right)\end{array}$ \\
\hline NGC 6528 & 0.62 & 0.23 : & $0.23:$ & 0.17 & 0.12 & 0.64 & 0.63 \\
\hline NGC 5927 & 0.46 & 0.13 & 0.13 & 0.08 & $\leqslant 0.05$ & 0.55 & 0.48 \\
\hline NGC 6624 & 0.28 & 0.08 & 0.08 & 0.08 & 0.10 & 0.25 & 0.22 \\
\hline NGC 6171 & 0.33 & 0.13 & 0.13 & 0.05 & $\leqslant 0.05$ & 0.33 & 0.25 \\
\hline NGC 7099 & 0.05 & $\leqslant 0.05$ & $\leqslant 0.05$ & $\leqslant 0.08$ & $\leqslant 0.08$ & $\leqslant 0.10$ & $\leqslant 0.10$ \\
\hline
\end{tabular}

TABLE 9. Predicted versus observed ratios for color widths.

\begin{tabular}{lcccccc}
\hline \hline Cluster & $\begin{array}{c}\mathrm{E}(B-V) \\
(\mathrm{mag})\end{array}$ & $\frac{\delta(V-K)}{\delta(J-K)}$ & $\begin{array}{c}\text { Allowed } \\
\text { Range }\end{array}$ & $\frac{\delta(V-K)}{\delta(B-V)}$ & $\begin{array}{c}\text { Allowed } \\
\text { Range }\end{array}$ & $\frac{\delta[E(B-V)]}{E(B-V)}$ \\
\hline NGC 6528 & 0.62 & 4.3 & $(3.1,6.3)$ & 2.8 & $(2.2,3.6)$ & 0.37 \\
NGC 5927 & 0.46 & 8.5 & $(5.2,18.3)$ & 3.9 & $(2.9,5.5)$ & 0.40 \\
NGC 6624 & 0.28 & 2.7 & $(1.7,4.7)$ & 3.0 & $(1.8,5.6)$ & 0.31 \\
NGC 6171 & 0.33 & 7.3 & $(4.2,16.5)$ & 2.2 & $(1.6,3.3)$ & 0.31 \\
Expected: & & & & & & \\
$\delta(B-V)$ & & 5.4 & & 8.6 & & \\
$\delta[\mathrm{Fe} / \mathrm{H}]$ & & 4.1 & & & & \\
\hline \hline
\end{tabular}

TABLE 10. Mean colors of giant branches of globular clusters.

\begin{tabular}{|c|c|c|c|c|c|c|c|}
\hline Cluster & $\begin{array}{c}\mathrm{E}(B-V) \\
(\mathrm{mag})\end{array}$ & $\begin{array}{c}{[\mathrm{Fe} / \mathrm{H}]} \\
(\mathrm{dex})\end{array}$ & $\begin{array}{c}\text { Distance } \\
\text { Modulus (mag) }\end{array}$ & $\begin{array}{c}\left\langle(J-K)_{0}\right\rangle \\
\left(M_{K_{0}}=-4\right)\end{array}$ & $\begin{array}{c}\left\langle(J-K)_{0}\right\rangle \\
\left(M_{K_{0}}=-5\right)\end{array}$ & $\begin{array}{c}\left\langle(V-K)_{0}\right\rangle \\
\left(M_{K_{0}}=-4\right)\end{array}$ & $\begin{array}{c}\left\langle(V-K)_{0}\right\rangle \\
\left(M_{K_{0}}=-5\right)\end{array}$ \\
\hline NGC 6528 & 0.55 & -0.23 & 14.42 & 0.92 & 1.03 & 3.85 & 4.62 \\
\hline NGC 5927 & 0.46 & -0.31 & 14.17 & 0.82 & 0.92 & 3.27 & 3.88 \\
\hline NGC 6624 & 0.28 & -0.37 & 14.18 & 0.98 & 1.05 & 3.21 & 3.73 \\
\hline NGC 6171 & 0.33 & -0.99 & 13.81 & 0.85 & 0.96 & 2.98 & 3.46 \\
\hline NGC 7099 & 0.05 & -2.13 & 14.29 & 0.68 & 0.75 & 2.57 & 2.86 \\
\hline \multicolumn{8}{|c|}{ Additional Cluster: } \\
\hline NGC 6553 & 0.78 & -0.28 & 13.16 & 0.89 & 1.03 & 3.60 & 4.63 \\
\hline \multicolumn{8}{|c|}{ Calibrating Clusters: } \\
\hline 47 Tuc & 0.04 & -0.71 & 13.03 & 0.85 & 0.94 & 3.26 & 3.78 \\
\hline M13 & 0.02 & -1.65 & 14.13 & 0.70 & 0.79 & 2.70 & 3.08 \\
\hline M92 & 0.02 & -2.24 & 14.33 & 0.67 & 0.74 & 2.57 & 2.83 \\
\hline
\end{tabular}

TABLE 11. Abundances of metal rich globular clusters.

\begin{tabular}{lccccc}
\hline \hline Cluster & $\begin{array}{c}{[\mathrm{Fe} / \mathrm{H}]_{\mathrm{Z}}} \\
(\mathrm{dex})\end{array}$ & $\begin{array}{c}{[\mathrm{Fe} / \mathrm{H}]_{\mathrm{AZ}}} \\
(\mathrm{dex})\end{array}$ & $\begin{array}{c}{[\mathrm{Fe} / \mathrm{H}]_{\mathrm{C}}} \\
(\mathrm{dex})\end{array}$ & $\begin{array}{c}{[\mathrm{Fe} / \mathrm{H}]_{\mathrm{FCP}}} \\
(\mathrm{dex})\end{array}$ & $\begin{array}{c}{[\mathrm{Fe} / \mathrm{H}]_{\mathrm{CS}}} \\
(\mathrm{dex})\end{array}$ \\
\hline NGC 6528 & -0.04 & -0.25 & $\ldots$ & $\ldots$ & +0.10 \\
NGC 5927 & -0.23 & -0.30 & -0.12 & -0.30 & -0.66 \\
NGC 6624 & -0.31 & -0.30 & $\ldots$ & $\ldots$ & -0.58 \\
NGC 6171 & -0.96 & $\ldots$ & -0.93 & -1.04 & -1.00 \\
NGC 7099 & -2.26 & $\ldots$ & $\ldots$ & $\ldots$ & -2.15 \\
Additional Cluster: & & & & \\
NGC 6553 & -0.03 & $\ldots$ & -0.31 & $(-0.30)$ & +0.10 \\
\hline \hline
\end{tabular}

Normalized to $[\mathrm{Fe} / \mathrm{H}]_{47 \text { Tuc }}=-0.71 \mathrm{dex}$ 
6553 appear to be too metal rich, while NGC 5927 and NGC 6624 appear too metal poor, relative to 47 Tuc. We remark that Kuchinski et al. (1994) have also found that NGC 5927 in the infrared is essentially identical to 47 Tuc, and is not much more metal-rich than 47 Tuc.

The measurements of integrated light spectral features are insensitive to choices of distance or reddening, while those based on giant branch properties or on spectroscopic analysis of individual stars do depend on the choice for these parameters. The horizontal branch apparent $V$ magnitudes are well determined, even for these crowded and metal rich clusters in very crowded fields, from our new Tololo photometry or from Ortolani et al. for NGC 6528, and no error larger than $0.10 \mathrm{mag}$ is allowed. Numerical tests indicate that if the true distance modulus is overestimated by $0.4 \mathrm{mag}$, the deduced abundance of the globular cluster from the infrared colors of its giant branch is underestimated by 0.35 to $0.55 \mathrm{dex}$. Since the allowed errors in the horizontal branch apparent magnitude are much smaller than $0.4 \mathrm{mag}$, this effect is probably not contributing in a significant way. Similarly, changing the adopted $M_{V}$ calibration for the RR Lyraes to any other plausible choice will not do much to the deduced abundances, since everything is measured differentially with respect to the calibrating clusters.

Different choices for the interstellar reddening will not only affect the giant branch colors directly, but will also affect the deduced distance modulus. Numerical tests of this case indicate that if $\mathrm{E}(B-V)$ is reduced by $0.10 \mathrm{mag}$, and the distance modulus adjusted appropriately, the metallicity of the globular cluster as inferred from the colors of its upper giant branch stars is increased by 0.16 dex.

Another potential cause of uncertainty is the absolute calibration of our infrared photometry. By comparison of our measurements with previously published and recent unpublished infrared photometry, we have shown above that these errors do not exceed $0.10 \mathrm{mag}$ at $K$. This translates into $\Delta[\mathrm{Fe} / \mathrm{H}] \approx 0.12 \mathrm{dex}$ for a very metal rich globular cluster, which is smaller than the discrepancies in abundance that we are discussing.

One might therefore suspect our small problems with NGC 5927 and NGC 6624 to be caused by a combination of the allowable small error in apparent magnitude of the horizontal branch $(0.10 \mathrm{mag})$ and the distance modulus. One might also try slightly decreasing the reddening, although that of NGC 6624 is so small already $[\mathrm{E}(B-V)=0.31 \mathrm{mag}]$ that no significant decrease seems plausible. The extrapolation we have used of the giant branch color changes with metallicity beyond 47 Tuc to such metal rich objects as NGC 6528 and NGC 6553 is such that the metallicity of those two objects has probably been slightly overestimated.
There is no completely satisfactory solution for reproducing from our infrared photometry the previously determined metallicities for these three globular clusters.

We summarize our conclusions as follows:

(1) The ordering of the metallicities we have derived for the observed clusters is correct, but there are some problems with the detailed numerical values. NGC 5927 and NGC 6624 appear to be more metal poor, and much closer to 47 Tuc in metallicity, than was previously believed. On the other hand, if our adopted value for the gradient $\Delta[\mathrm{Fe} / \mathrm{H}] / \Delta(V-K)_{0}$ is correct (and recall that we were forced to extrapolate in color considerably redder than the giant branch of $47 \mathrm{Tuc}$, the most metal rich calibrating cluster), then NGC 6528 and NGC 6553 may be more metal rich than was previously believed.

(2) A spread in the giant branch of $\delta(V-K) \approx 0.5 \mathrm{mag}$ at a fixed $K$ magnitude is seen in NGC 5927. A spread of about $0.6 \mathrm{mag}$ is seen in NGC 6528. If these were due solely to differential reddening, $\delta \mathrm{E}(B-V)$ would be about 0.20 mag in NGC 5927 and about 0.24 mag in NGC 6528. (In NGC 5927, a deliberate effort was made to observe in an area found by previous optical studies to have relatively uniform absorption.) The range of $V-K$ is smaller in the other clusters observed.

These numbers are in good agreement with estimates from previous optical studies of the magnitude of the differential reddening within the clusters.

(3) The observed spread in various colors along the giant branch for the heavily reddened globular clusters in our sample is consistent with patchy interstellar reddening strongly varying on a small spatial scale. No abundance variations within clusters are required to match the observations.

(4) The observed spreads of the upper giant branch in these metal rich globular clusters at various colors confine the internal range of metallicity. This must be less than 0.2 dex in NGC 5927 and less than 0.1 dex in NGC 7099. The spread in $V-K$ color at a fixed $K$ magnitude is a strong constraint on $[\mathrm{Fe} / \mathrm{H}]$ variations within a cluster.

Eric Persson and his team built the infrared camera in use at the Las Campanas Observatory. We are grateful to him and to Jay Frogel for several useful discussions. We are grateful to the Cerro Tololo Inter-American Observatory for the service observing of CCD images in $V$ and $R$ of NGC 5927 and of NGC 6624 using the Yale telescope. Keith Matthews helped with the infrared observations at the $200 \mathrm{in}$. telescope at Palomar Mountain. ECS was partially supported by a stipend from the National Physical Science Consortium.

\section{REFERENCES}

Alcaino, G., \& Liller, W. M. H. 1980, AJ, 85, 1330

Armandroff, T. E., \& Zinn, R. 1988, AJ, 96, 92

Bolte, M. 1987, ApJ, 319, 760

Cohen, J. G. 1983, ApJ, 270, 654

Cohen, J. G. 1992, ApJ, 400, 528

Cohen, J. G., Frogel, J. A., \& Persson, S. E. 1978, ApJ, 222, 165
DaCosta, G. S., Mould, J. R., \& Ortolani, S. 1984, ApJ, 282, 125

Davidge, T. J., Harris, W. E., Bridges, T. J., \& Hanes, D. A. 1992, ApJS, 81, 251

Davidge, T. J., \& Simons, D. A. 1991, AJ, 101, 1720

Davidge, T. J., \& Simons, D. A. 1994, AJ, 107, 240

Dickens, R. J. 1972, MNRAS, 157, 299 
Elias, J. H., Frogel, J. A., Matthews, K., \& Neugebauer, G. 1982, AJ, 87, 1029

Frogel, J. A., Cohen, J. G., \& Persson, S. E. 1983a, ApJ, 275, 773

Frogel, J. A., Persson, S. E., \& Cohen, J. G. 1981, ApJ, 246, 842

Frogel, J. A., Persson, S. E., \& Cohen, J. G. 1983b, ApJS, 53, 713

Green, E. M., Demarque, P., \& King, C. R. 1987, The Revised Yale Isochrones and Luminosity Functions (Yale University Observatory)

Hartwick, F. D. A. 1975, PASP, 87, 77

Jones, R. V., Carney, B. W., Storm, J., \& Latham, D. W. 1992, ApJ, 386, 646

Kuchinski, L., Frogel, J. A. F., Terndrop, D., \& Persson, S. E. 1994 (in preparation)

Liu, T. X., \& Janes, K. A. 1990, ApJ, 360, 561

Liller, M. H., \& Carney, B. W. 1978, ApJ, 224, 383
Menzies, J. 1974, MNRAS, 169, 79

Ortolani, S., Barbuy, B., \& Bica, E. 1991a, ApJ, 249, L31

Ortolani, S., Bica, E., \& Barbuy, B. 1991b, A\&AS, 92, 441

Persson, S. E., West, S. C., Carr, D. M., Sivaramakrishnan, A., \& Murphy, D. C. 1992, PASP, 104, 204

Peterson, C. 1993, in The Structure and Dynamics of Globular Clusters, edited by S. Djorgovski and G. Meylan (ASP)

Sandage, A. R., \& Katem, B. 1964, ApJ, 139, 1088

Sawyer Hogg, H. 1973, Publications of the David Dunlop Observatory, 3

Stetson, P. B. 1987, PASP, 99, 99, 191

van den Bergh, S., \& Younger, F. 1979, AJ, 84, 1305

Zinn, R. J. 1980, ApJS, 42, 19

Zinn, R., \& West, M. 1984, ApJS, 55, 45 